\title{
Characterization of Convex and Concave Resource Allocation Problems in Interference Coupled Wireless Systems
}

\author{
Holger Boche, Fellow, IEEE, Siddharth Naik, and Tansu Alpcan, Member, IEEE
}

\begin{abstract}
This paper investigates the possibility of having convex or concave formulations of optimization problems for interference coupled wireless systems. An axiomatic framework for interference functions proposed by Yates in 1995 is used to model interference coupling in our paper. The paper shows that under certain natural assumptions, the exponential transformation is the unique transformation (up to a positive constant) for "convexification" of resource allocation problems for linear interference functions. Furthermore, it is shown that under certain intuitive assumptions, it is sufficient to check for the joint concavity (convexity) of sum of weighted functions of SINR (inverse SINR) with respect to $s$ ( $p=e^{s}$, where $p$ is the power vector of the users), if we would like the resulting resource allocation problem to be concave (convex). This paper characterizes the largest class of utility functions and the largest class of interference functions (respectively), which allow a convex and concave formulation of a problem for interference coupled wireless systems. It extends previous literature on log-convex interference functions and provides boundaries on the class of problems in wireless systems, which can be algorithmically tackled by convex optimization techniques.
\end{abstract}

Index Terms-Concavity, convexity, exponential transformation, interference coupled systems, resource allocation.

\section{INTRODUCTION}

$\mathbf{T}$ HERE is fair amount of general consensus, that it is possible to classify problems into certain "categories" of being solvable based on their convexity properties [1], [2]. In our paper we check for the joint (convexity) concavity of functions, which are functions of the (inverse) signal-to-interference (plus noise) ratio (SINR), which is an important measure for link performance in wireless systems. Our paper focuses on the topic of investigating convexity properties of functions of inverse SINR and concavity properties of functions of SINR. Such functions are frequently encountered as loss minimization problems in wireless communications, e.g.

Manuscript received July 05, 2010; revised November 01, 2010; accepted January 05, 2011. Date of publication February 10, 2011; date of current version April 13, 2011. The associate editor coordinating the review of this manuscript and approving it for publication was Prof. Wolfgang Utschick. This work was supported in part by the project Spectrum Allocation Games (SAGa) in cooperation with Deutsche Telekom Laboratories. The work of S. Naik was supported in part by the DFG Project BO 1734/23-1 and the DFG Project BO 1734/24-1.

H. Boche and S. Naik were with the Heinrich Hertz Institute (HHI) and the Technical University of Berlin, Germany. The are now with the Institute for Theoretical Information Technology, Technical University of Berlin, $80331 \mathrm{Mu}$ nich, Germany (e-mail: boche@tum.de; sid.naik@tum.de).

T. Alpcan is with the Technical University of Berlin, Deutsche Telekom Laboratories, 10587 Berlin, Germany (e-mail: alpcan@sec.t-labs.tu-berlin.de).

Digital Object Identifier 10.1109/TSP.2011.2112652 minimum mean-square error (MMSE) and bit error rate (BER). They are also encountered as utility maximization problems, e.g. rate and capacity.

Wireless communication systems are often interference coupled. We adopt an axiomatic approach to capture interference coupling. An axiomatic approach was proposed by Yates in [3] with extensions in [4]-[7]. The Yates framework of standard interference functions (discussed in Section II-B) is general enough to incorporate cross-layer effects and it serves as a theoretical basis for a many algorithms.

Certain examples, where the interference function framework has been utilized are as follows: beamforming [8]-[10], CDMA [11], base station assignment, robust design [12], [13], transmitter optimization [14], [15], and characterization of the Pareto boundary [16]. The framework can be used to combine power control [17] and adaptive receiver strategies. In [18], it was proposed to incorporate admission control to avoid unfavorable interference scenarios. In [19], it was proposed to adapt the quality of service (QoS) requirements to certain network conditions. In [20], a power control algorithm using fixed-point iterations was proposed for a modified cost function, which permits control of convergence behavior by adjusting fixed weighting parameters.

Proving inherent boundaries on the problems, which can be characterized as jointly convex problems could help in channelizing future research directions and help obtain practically implementable resource allocation strategies utilizing the wide gamut of convex optimization tools. We focus our attention on a problem, namely that of characterizing the subclass of general interference functions for which we can get a meaningful convex optimization problem from a wireless systems perspective. Solving problems with real time constraints is a critical issue in current wireless systems. In general, interference coordination and management is an important research topic and has potential to address problems in future generations of wireless systems, e.g., indoor interference problem, possibility to enhance capacity by utilizing interference positively via relaying in overlay cognitive radio systems. The papers [21] and [22] discuss the structure and modeling of interference via interference functions. References [21] and [22] essentially focus on the properties of interference functions and characterization of interference coupling in wireless systems. The paper [23] proves that there exists no SINR based utility functions, which are convex or concave in the power domain. Furthermore, [23] showed, that the weighted sum of such functions can never be convex or concave in the power domain. 
Hence, we investigate for possible transformations to other domains to exploit hidden convexity and concavity properties, respectively. One such transformation is the exponential transformation. Such an exponential transformation has been utilized earlier in [24]-[29]. References [30] and [31] exploit hidden convexity in the context of utility maximization using geometric programming. In [32], a majorization technique is used to compute the optimal solution over a logarithmic utility function. In [33], hidden convexity is exploited to provide robust resource allocation and certain distributed implementation have been provided.

Such a change of variable is an additional motivation for the utilization of the log-domain in communication systems. Our list is by no means comprehensive and the reader is requested to refer to the listed papers and the references therein for further literature on the topic. We differentiate ourselves from previous work by checking the following: let a transformation to exploit hidden convexity or concavity properties exist. Then, is this transformation unique? Furthermore, we search for the largest class of utility functions and the largest class of interference functions, which permit such transformations to exploit hidden convexity. This paper sets limitations on the class of utility functions, types of interference coupling in wireless systems and in turn resource allocation problems, which can have desirable concavity and convexity properties. Problems outside these characterized classes of functions can never be transformed into suitable concave or convex characterizations.

\section{Problem Statement AND CONTRIBUtions}

In this section, we precisely state the problems addressed in the paper and follow it up with the main contributions of the paper. We begin by providing certain notational conventions used in our paper in Section II-A below.

\section{A. Preliminaries and Notation}

Matrices and vectors are denoted by bold capital letters and bold lowercase letters, respectively. Let $Y$ represent a matrix. Let $\operatorname{diag}\left\{x_{1}, \ldots, x_{K}\right\}$ represent a matrix with $x_{1}, \ldots, x_{k}$ in the diagonal positions, respectively and zero entries in all other positions. Let $\boldsymbol{y}$ be a vector, then $y_{l}=[\boldsymbol{y}]_{l}$ is the $l^{\text {th }}$ component. The notation $y \geq 0$ implies that $y_{l} \geq 0$ for all components $l$. $\boldsymbol{x}>\boldsymbol{y}$ implies $x_{l}>y_{l}$ for all components $l$. Similar definitions hold for the reverse directions. $\boldsymbol{x} \neq \boldsymbol{y}$ implies that the vector differs in at least one component. Let $\boldsymbol{y}^{T}$ represent a transpose of the vector $y$. Let calligraphic letters, i.e., $\mathcal{F}$ imply a set, with the exception that $\mathcal{I}$ is used to represent a function. Let $\mathcal{F}_{1} \subsetneq \mathcal{F}_{2}$ imply that $\mathcal{F}_{1}$ is a proper subset of $\mathcal{F}_{2}$. The set of real numbers is denoted as $\mathbb{R}$. The set of non-negative real numbers is denoted as $\mathbb{R}_{+}$. The set of positive real numbers is denoted as $\mathbb{R}_{++}$. Let $e^{\boldsymbol{y}}$ and $\log (\boldsymbol{y})$ denote component-wise exponential and logarithm, respectively. The symbols will represent $g, f, h$, and $\psi$ functions throughout the paper.

\section{B. Interference Functions}

In a wireless system, the utilities of the users can strongly depend on the underlying physical layer. An important measure for the link performance is the SINR ratio. Consider $K$ users with transmit powers $\boldsymbol{p}=\left[p_{1}, \ldots, p_{K}\right]^{T}$ and $\mathcal{K}:=\{1, \ldots, K\}$.
The noise power at each receiver is $\sigma^{2}$. Hence, the SINR at each receiver depends on the extended power vector $\boldsymbol{p}$ where $\underline{\boldsymbol{p}}=\left[\boldsymbol{p}, \sigma^{2}\right]^{T}=\left[p_{1}, \ldots, p_{K}, \sigma^{2}\right]^{T}$. The resulting SINR of user $\bar{k}$ is

$$
\operatorname{SINR}_{k}(\underline{\boldsymbol{p}})=\frac{p_{k}}{\mathcal{I}_{k}(\underline{\boldsymbol{p}})}=\gamma_{k}(\underline{\boldsymbol{p}})
$$

where $\mathcal{I}_{k}$ is the interference (plus noise) as a function of $\boldsymbol{p}$. In order to model interference coupling, we shall follow the axiomatic approach proposed in [3] and [7]. The general interference functions possess the properties of conditional positivity $(A 1)$, scale invariance $(A 2)$ and monotonicity with respect to the power component $(A 3)$, and strict monotonicity with respect to the noise component $(A 4)$. For further details, kindly refer to the Appendix. We will also utilize the class of log-convex interference functions, which we introduce below. Log-convexity is a useful property that allows one to apply convex optimization techniques to certain non-convex problems.

Definition 1: Log-convex interference function: An interference function $\mathcal{I}: \mathbb{R}_{+}^{K+1} \mapsto \mathbb{R}_{+}$is said to be a log-convex interference function if $A 1-A 4$ are fulfilled and $\mathcal{I}(\exp \{\boldsymbol{s}\})$ is log-convex on $\mathbb{R}^{K+1}$.

Let $f(\boldsymbol{s}):=\mathcal{I}(\exp \{\boldsymbol{s}\})$. A function $f: \mathbb{R}^{K+1} \mapsto \mathbb{R}_{+}$is log-convex on $\mathbb{R}^{K+1}$, if and only if $\log f$ is convex or equivalently $f(\boldsymbol{s}(\lambda)) \leq f\left(\boldsymbol{s}^{(1)}\right)^{1-\lambda} f\left(\boldsymbol{s}^{(2)}\right)^{\lambda}$, for all $\lambda \in(0,1)$, $\boldsymbol{s}^{(1)}, \boldsymbol{s}^{(2)} \in \mathbb{R}^{K+1}$, where $\boldsymbol{s}(\lambda)=(1-\lambda) \boldsymbol{s}^{(1)}+\lambda \boldsymbol{s}^{(2)}, \lambda \in$ $(0,1)$. Note that the log-convexity in Definition 1 is based on a change of variable $\underline{p}=\exp \{\boldsymbol{s}\}$ (component-wise exponential). Such a technique has been previously used to exploit a "hidden convexity" of functions [24]-[27], which are otherwise non-convex. It was also utilized in a different context in [28].

\section{Impact of Interference Coupling}

Users in a wireless systems coupled by interference are intrinsically competitive. Each of them is principally interested in maximizing their own utility. Such a characterization is accompanied by a precondition that there must be at least one user $k \in \mathcal{K}$ who sees interference from another user $j \in \mathcal{K}$ and $j \neq k$, i.e., it must not be possible to completely orthogonalize all the users in the system. If the users are completely orthogonalized, then they are coupled only by the constraints on the resource allocation strategy and there is no "competition" in the sense as we describe in this section. The example below highlights this point and displays the impact of interference coupling. Let $u_{k}$ represent an utility function corresponding to a user $k$, where $k \in \mathcal{K}$.

Example 1: Consider the utility function $u_{k}(\underline{\boldsymbol{p}})=$ $\log \left(p_{k} / \mathcal{I}_{k}(\boldsymbol{p})\right)$. The function

$$
f(\underline{\boldsymbol{p}}, \boldsymbol{\omega})=\sum_{k \in \mathcal{K}} \omega_{k} u_{k}(\underline{\boldsymbol{p}})
$$

for all weight vectors $\boldsymbol{\omega}>\mathbf{0}$ is never jointly concave with respect to $\boldsymbol{p}$. Furthermore the function (2) is not a convex optimization problem even for linear interference functions, e.g. $\mathcal{I}_{k}(\underline{\boldsymbol{p}})=\sum_{l \in \mathcal{K}} v_{k l} p_{\boldsymbol{l}}+\sigma_{k}^{2}$, where $v_{k l}$ is the link-gain between 
transmitter $l$ and receiver $k$. This holds for all non-orthogonal system of users, i.e., there exists at least one $l$ such that $v_{k l} \neq 0$ for $k \neq l$, i.e., each users sees at least one other user as interference.

Similarly, the problem of minimizing the function $f(\underline{\boldsymbol{p}}, \boldsymbol{\omega})=$ $\sum_{k \in \mathcal{K}} \omega_{k} \log \left(\frac{\mathcal{I}_{k}(\underline{\boldsymbol{p}})}{p_{k}}\right)$ for all weight vectors $\boldsymbol{\omega}>\mathbf{0}$ is not jointly convex with respect to $\boldsymbol{p}$.

Reference [23] shows that if $u_{k}$ is the rate of user $k$, then the following sum of weighted rate maximization problem cannot be jointly concave in its current form. Similarly, if we have that $u_{k}$ is the minimum-mean-square-error (MMSE) of user $k$, then the following sum of weighted MMSE minimization problem cannot be jointly convex in its current form. None the less, through appropriate substitution of variables, the above formulation can be converted into a convex or concave optimization problem.

\section{Problem Statement}

Based on the observations from Section II-C, the following important questions arise immediately.

Problem 1: Can the function $\frac{\mathcal{I}_{k}(\psi(\boldsymbol{s}))}{\psi\left(\boldsymbol{s}_{k}\right)}$ be jointly convex after a suitable transformation (where $\boldsymbol{p}=\psi(\boldsymbol{s})$ is a transformation) for linear interference functions?

1) Under what conditions is such a transformation unique?

2) Can these results be extended beyond linear interference functions?

Certain examples of linear interference functions beyond the case of linear interference functions are as follows:

- convex interference functions: interference functions utilized to model worst-case models, e.g.

$$
\mathcal{I}_{k}(\boldsymbol{p})=\max _{c \in \mathcal{X}}[\boldsymbol{V}(c) \boldsymbol{p}]_{k}, \quad \forall k \in \mathcal{K}
$$

where the parameter $c$, chosen from a closed bounded set $\mathcal{X}$ can stand for the impact of error effects. Examples of error effects could be channel estimation and prediction errors. Performing power allocation with respect to worst case interference such as (3) guarantees a certain degree of robustness (see, e.g. [10] and references therein.) and

- concave interference functions: interference functions representing interference coupling in uplink beamforming with $K$ single-antenna transmitters and an $M$-element antenna array at the receiver [22, Sec. I-A and Sec. II].

There are many such examples frequently encountered in practical wireless systems The Problem 1 has been formulated for the convex case. We can formulate a similar problem for the concave case as follows:

Problem 2: Can the function $\frac{\psi\left(s_{k}\right)}{\mathcal{I}_{k}(\psi(\boldsymbol{s}))}$ be jointly concave after a suitable transformation for linear interference functions?

1) Under what conditions is such a transformation unique?

2) Can these results be extended beyond linear interference functions?

In Problems 1 and 2, we have taken the perspective of an arbitrary user $k \in \mathcal{K}$. To address these problems, we shall formulate certain requirements. For formulating these requirements, we shall briefly review the concepts of feasible SINR regions and feasible quality-of-service (QoS) regions. The feasible SINR region $\mathcal{F}$ is the set of all feasible SINR vectors $\hat{\gamma}$, that can be supported for all users by means of power control, with interference being treated as noise. We define a set $\mathcal{P}$ as the set of vectors, which satisfy certain power constraints, e.g.,

- for the case of total power constraints: $\mathcal{P}:=\{\boldsymbol{p} \mid \boldsymbol{p}=$ $\left.\left[p_{1}, \ldots, p_{K}\right], \sum_{k \in \mathcal{K}} p_{k} \leq P_{\text {total }}\right\}$, where $P_{\text {total }}$ is the total power constraint;

- for the case of individual power constraints: $\mathcal{P}:=\{\boldsymbol{p} \mid \boldsymbol{p} \leq$ $\hat{\boldsymbol{p}}\}$, where $\hat{\boldsymbol{p}}=\left[\hat{p}_{1}, \ldots, \hat{p}_{K}\right]$ are the individual power constraints; and

- for the case of individual and total power constraints: $\mathcal{P}:=$ $\left\{\boldsymbol{p} \mid \boldsymbol{p}=\left[p_{1}, \ldots, p_{K}\right], \sum_{k \in \mathcal{K}} p_{k} \leq P_{\text {total }}, \boldsymbol{p} \leq \hat{\boldsymbol{p}}\right\}$.

The feasible SINR region $\mathcal{F}$ can be written as follows:

$$
\mathcal{F}=\left\{\hat{\gamma} \mid \exists \underline{p} \geq \mathbf{0}, \boldsymbol{p} \in \mathcal{P}, \gamma_{k}(\underline{\boldsymbol{p}}) \geq \hat{\gamma}_{k}, \hat{\gamma}_{k} \in \mathbb{R}_{+}, \forall k \in \mathcal{K}\right\}
$$

and the corresponding feasible QoS region is

$$
\mathcal{U}=\left\{\hat{\boldsymbol{u}} \mid \exists \boldsymbol{\gamma} \in \mathcal{F}, u_{k}\left(\gamma_{k}(\underline{\boldsymbol{p}})\right) \geq \hat{u}_{k}, \hat{u}_{k} \in \mathbb{R}_{+}, \forall k \in \mathcal{K}\right\}
$$

Example 2: Consider the function $\log \left(\frac{p_{2}}{p_{1}+\sigma^{2}}\right)$, which is concave with respect to $p_{2}$ and convex with respect to $p_{1}$. The function $\log \left(\frac{p_{2}}{p_{1}+\sigma^{2}}\right)$ in neither jointly convex nor jointly concave with respect to $\boldsymbol{p}=\left[p_{1}, p_{2}\right]^{T}$.

We know from [34] and the references there in, that the feasible $\operatorname{SINR}$ region $(\mathcal{F})$ is in general not convex.

Example 3: Consider an example with two users, where the SINR of user 1 is $\gamma_{1}(\underline{\boldsymbol{p}})=\frac{p_{1}}{v_{11} p_{1}+v_{12} p_{2}+\sigma^{2}}$ and the SINR of user 2 is $\gamma_{2}(\underline{\boldsymbol{p}})=\frac{p_{2}}{v_{21} p_{1}+v_{22} p_{2}+\sigma^{2}}$, where $v_{k j}$ is the channel gain between the $k^{\text {th }}$ receiver and $j^{\text {th }}$ transmitter.

The feasible signal-to-interference ratio (SIR) region can also be defined as $\{\boldsymbol{\gamma}>\mathbf{0} \mid \rho(\boldsymbol{\gamma}) \leq 1\}$, where $\rho(\boldsymbol{\gamma}):=\rho\left(\operatorname{diag}\{\boldsymbol{\gamma}\} \boldsymbol{V}_{\text {res }}\right)$ is the Perron root of the weighted coupling matrix, where $\boldsymbol{V}=\left[\boldsymbol{V}_{\text {res }}, \mathbf{1}^{T}\right]$ and $\boldsymbol{V}_{\text {res }}$ is a $K \times K$ restricted weighted coupling matrix containing the interference coupling coefficients (without the dependency on noise). Furthermore, we also know from [23], that we can never have joint convexity of the inverse SINR in the power domain. Hence, we would like to investigate the possibility of finding a suitable transformation $\psi$ (or $\left.\psi^{-1}\right)$, which:

1) transforms the problem from the power domain to the $\boldsymbol{s}$-domain, i.e., $\psi^{-1}: \mathbb{R}_{+} \mapsto S$, where $S=\mathbb{R}, s \in S$ and the inverse SINR and functions of inverse SINR are jointly convex with respect to $\boldsymbol{s}=\left[s_{1}, \ldots, s_{K}\right]$;

2) transforms the feasible SINR region into a convex feasible QoS set $\mathcal{U}$, where $\psi^{-1}\left(\gamma_{k}\right)=u_{k}$, for all $k \in \mathcal{K}$ and $\boldsymbol{u} \in \mathcal{U}$. While looking for our transformation $\psi^{-1}$, we make the following assumption: Transformation $\psi(s)=p$ is strictly monotonic increasing and twice continuously differentiable throughout the paper.

The feasible SIR region and the feasible SINR region are convex after the transformation $\log (1 / \mathrm{SIR})$ and $\log (1 / \mathrm{SINR})$, respectively (see Fig. 1 for an example of the convexity of the transformed SINR region). The convexity of the feasible SINR region is a direct consequence of the Perron root 


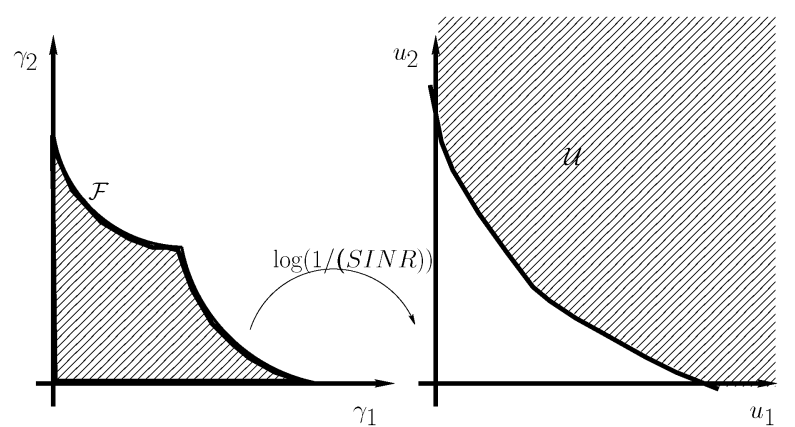

Fig. 1. Depiction of a feasible SINR region $\mathcal{F}$ for individual power constraints and the corresponding feasible QoS region $\mathcal{U}$ after a transformation $\log (1 / \mathrm{SINR})$.

$\rho(\boldsymbol{\gamma})$ being log-convex after a change of variable $\boldsymbol{\gamma}=e^{\boldsymbol{s}}$, where $\boldsymbol{s} \in \mathbb{R}^{K}$ is the logarithmic SIR. Note that $\rho(\boldsymbol{\gamma})$ fulfills the axioms $A 1$ to $A 4$, i.e., the Perron root is a special case of the more general interference functions. This does not imply that $\log \frac{v_{11} p_{1}+v_{12} p_{2}+\sigma^{2}}{p_{1}}$ is jointly convex, nor that $\omega_{1} \log \frac{v_{11} p_{1}+v_{12} p_{12}+\sigma^{2}}{p_{1}}+\omega_{2} \log \frac{v_{21} p_{1}+v_{22} p_{12}+\sigma^{2}}{p_{2}}$ is jointly convex, where $\left[\omega_{1}, \omega_{2}\right]=\boldsymbol{\omega} \geq \mathbf{0}$.

Remark 1: Even though the feasible QoS region $\mathcal{U}$ is a convex set (see Fig. 1) after a logarithmic transformation, the function $\log \left(1 / \gamma_{k}(\underline{\boldsymbol{p}})\right)$, for $k \in \mathcal{K}$ is not jointly convex with respect to $\underline{\boldsymbol{p}}$.

Linear interference functions are the simplest type of interference functions and they are frequently encountered in communication systems. Hence, expecting the feasible QoS region $\mathcal{U}$ to be convex (see condition 2 above, before the introduction of the transformation $\psi$ ) for all linear interference functions, is a natural requirement for communication systems.

We now return to the problem of finding a suitable transformation $\psi$. Let $\boldsymbol{\Gamma}:=\operatorname{diag}\left\{\gamma_{1}, \ldots, \gamma_{K}\right\}$, where $\gamma_{k}=\psi\left(u_{k}\right)$. From [34] we know that, $\rho(\boldsymbol{\Gamma V})$ is convex for all coupling matrices $V \in \mathbb{R}_{+}^{K \times K}$ and for all users $k \in \mathcal{K}$, if and only if $\psi$ is log-convex. Furthermore, this implies that the feasible QoS region $\mathbf{U}$ resulting from the transformation $\gamma_{k}=\psi\left(u_{k}\right)$, for all users $k \in \mathcal{K}$ without power constraints is convex. Now, to formalize the conditions 1) and 2) we introduce the following requirement.

Requirement 1: For all linear interference functions, the $\rho(\Gamma V)$ is convex for all $\boldsymbol{V} \in \mathbb{R}_{+}^{K \times K}$ for all users $k \in \mathcal{K}$, where $\boldsymbol{V}$ is the link gain matrix.

Remark 2: If Requirement 1 is satisfied, we have that the feasible QoS region is convex for all linear interference functions. Then, from [34, Theorem 1] we have that $\psi$ is log-convex.

The function $\psi(s)=e^{s}$ is one such function satisfying Requirement 1. We further introduce another requirement, which expects joint convexity of the inverse SINR, which can be thought of as loss minimization in wireless systems and joint convexity of the inverse SINR raised to $\alpha$ for all $\alpha>0$.

Requirement 2: For all scalars $\alpha>0$ the function $\left(\frac{\mathcal{I}_{k}(\psi(\boldsymbol{s}))}{\psi\left(s_{k}\right)}\right)^{\alpha}$ is jointly convex with respect to $\boldsymbol{s}$.

Expecting the function $(1 / \mathrm{SINR})^{\alpha}$, with $\alpha>0$ to be convex with respect to $\boldsymbol{s}$, implies that we expect the expression of the $\alpha$-order diversity of a system with a certain inverse SINR to be convex. Reference [35] provides a characterization of the mul- tiplexing rate tuples of the users as a function of the common diversity gain for each user. It characterizes the diversity multiplexing trade-off in multiple access channels, when all users have the same diversity requirements. We now introduce the families of functions $\mathcal{C}$ onv and $\mathcal{E C} O n v$ below which will help us introduce our last requirement.

Definition 2: $\mathcal{C} o n v$ is the family of all strictly monotonic increasing, continuous and convex functions $g$. $\mathcal{E}$ Conv is the family of all strictly monotonic increasing, continuous function $g$, such that $g\left(e^{x}\right)$ is convex.

The inclusion order of the classes of utility sets is $\mathcal{C}$ onv $\subsetneq$ $\mathcal{E C}$ onv. In fact, $\mathcal{E C}$ onv is much larger the $\mathcal{C} o n v$. If a utility function $g$ is in the class $\mathcal{E C} o n v$, then it has the property that $g\left(e^{x}\right)$ is convex. The example $g(x)=\log x$, which is frequently encountered in wireless communication systems shows that even a concave function could be transformed into a convex function. Hence, we would like to investigate the possibility of ensuring convexity for the larger class of $\mathcal{E C}$ onv functions. For this purpose we introduce our last requirement, which expects joint convexity of functions of inverse SINR, which are frequently encountered in wireless systems, e.g. MMSE: $g(x)=1 /(1+x)$ and high-SNR approximation of BER $g(x)=x^{-\alpha}$ with diversity order $\alpha$.

We are now in a position to formulate the problems from a system level perspective, e.g. a weighted sum of minimum square error minimization problem from the perspective of a base station or a central controller.

Problem 3: Let $g_{1}, \ldots, g_{K}$ be strictly monotonic increasing, convex and continuous functions. Consider the function

$$
\sum_{k \in \mathcal{K}} \omega_{k} g_{k}\left(\frac{\mathcal{I}_{k}(\psi(\boldsymbol{s}))}{\psi\left(s_{k}\right)}\right) \text {. }
$$

1) For linear interference functions, can the function (6) be jointly convex after a suitable transformation?

2) Under what conditions is such a transformation unique?

3 ) If we relax the condition of linear interference functions, then for what kind of interference coupling can we extend the above results?

We now present our final requirement.

Requirement 3: For all functions $g_{k} \in \mathcal{E C}$ onv , the function (6) with $\sum_{k \in \mathcal{K}} \omega_{k}=1$ is jointly convex with respect to $\boldsymbol{s}$.

The function $\psi(s)=e^{s}$ is one such function satisfying Requirement 3.

We formulate a similar problem for the concave case. For this purpose we formally introduce two classes of utility functions.

Definition 3: $\mathcal{C}$ onc is the family of all strictly monotonic increasing, continuous and concave functions $g$. $\mathcal{E}$ onc is the family of all strictly monotonic increasing, continuous functions $g$, such that $g\left(e^{x}\right)$ is concave.

The concavity of the function $g\left(e^{y}\right)$ is a stronger requirement, i.e., $\mathcal{E C o n c} \subsetneq \mathcal{C}$ onc.

Problem 4: Let $g_{1}, \ldots, g_{K}$ be strictly monotonic increasing, concave and continuous functions. Consider the function

$$
\sum_{k \in \mathcal{K}} \omega_{k} g_{k}\left(\frac{\psi\left(s_{k}\right)}{\mathcal{I}_{k}(\psi(\boldsymbol{s}))}\right)
$$


1) For linear interference functions, can the function 7 be jointly concave after a suitable transformation?

2) Under what conditions is such a transformation unique?

3) If we relax the condition of linear interference functions, then for what kind of interference coupling can we extend the above results?

While answering the above problems in the paper, we shall have proved, that $\psi(x)=c \exp (\mu x)$, with $c, \mu>0$ is the only family of transformations, which satisfies our requirement. Hence, we shall utilize this transformation while analyzing problems 5 and 6.

Problem 5: Let us assume, that $\mathcal{I}_{1}, \ldots, \mathcal{I}_{K}$ are linear interference functions. What is the largest class of utility functions, i.e., functions $g_{1}, \ldots, g_{K}$, which are not necessarily convex, such that we can ensure the joint convexity of

$$
g_{k}\left(\frac{\mathcal{I}_{k}(\psi(\boldsymbol{s}))}{\psi\left(s_{k}\right)}\right) ?
$$

Problem 6: What is the largest possible class of interference functions, such that for all utility functions $g_{k} \in \mathcal{E C}$ onv, the function (6) is jointly convex with respect to $s$, for all weight vectors $\boldsymbol{\omega}>\mathbf{0}$.

\section{E. Contributions}

The main contributions of this paper are as follows:

- Linear interference functions are the simplest and most frequently encountered class of interference functions. Theorem 1 shows, that under certain natural assumptions for linear interference functions, the transformation $p_{k}=\exp \left(\mu s_{k}\right), \mu>0$ where $p_{k}$ is the power of an arbitrary user $k$ and $s \in \mathbb{R}$, is the unique transformation for "convexification" of resource allocation problems.

- If we would like the resulting resource allocation problem to be convex, then Theorems 1 and 3 show that under natural assumptions, it is sufficient to check for the joint convexity of the function (6) with respect to $\boldsymbol{s}$.

- Theorem 4 and Remark 8 extend the above analysis beyond linear interference functions. Theorem 4 and Remark 8 characterize the largest class of interference functions $(\mathcal{C}$ interference functions), which allow a problem in interference coupled wireless systems to be formulated as a convex optimization problem. $\mathcal{C}$ interference functions (see Definition 4), include log-convex interference functions, extending previous literature on the topic of convex characterization of resource allocation problems.

- Under certain natural assumptions, we present an impossibility result (Theorem 5), which states that there exists no transformation $\psi$, such that the function $\psi\left(s_{k}\right) / \mathcal{I}_{k}(\psi(\boldsymbol{s}))$ for all users $k$ is jointly concave with respect to $\boldsymbol{s}$.

- Theorem 7 establishes the largest class of utility functions $(\mathcal{E C}$ onc $)$, which are functions of SINR in the $\boldsymbol{s}$-domain and are concave. Due to a certain requirement of Theorem (explained in detail in Lemma 2), such a class of utility function is a restricted class. Furthermore, it is shown that the family of exponential functions is the unique family of functions, such that relevant and frequently encoun- tered functions in interference coupled wireless systems are jointly concave for all linear interference functions and for all utility functions in the class $\mathcal{E C}$ onc.

- Theorem 8 proves that the largest class of interference functions, which preserves concavity of resource allocation strategies of interference coupled wireless systems is the family of log-convex interference functions. Furthermore, it provided a complete characterization of the class of log-convex interference functions, with respect to convexity and concavity properties of resource allocation problems.

\section{AnAlysis of Resource Allocation Problems: CONVEX CASE}

We now analyze the convexity properties of functions of inverse SINR for linear interference functions.

\section{A. Analysis of Convexity Properties of Resource Allocation Problems for Linear Interference Functions}

We check for a transformation of the problem from the power domain to the $\boldsymbol{s}$-domain, with the hope that the resulting problem is convex, for all linear interference functions.

We now present a result, which shows that if we expect the supportable QoS region to be convex for all linear interference functions, then the only transformation (from the $s$-domain to power domain) permitted under certain conditions, is the family of exponential transformations (up to certain scalar $\mu$ ).

Theorem 1: Transformation $\psi$ satisfies Requirements 1 and 2 , if and only if there exists $a \mu, c>0$ such that $\psi\left(s_{k}\right)=$ $c \exp \left(\mu s_{k}\right), s \in \mathbb{R}$, for $1 \leq k \leq K+1$.

Proof: "£": This direction can be easily verified as follows. Let $\boldsymbol{s}(\lambda)=(1-\lambda) \boldsymbol{s}^{(1)}+\lambda \boldsymbol{s}^{(2)}$. Let $\mathcal{I}_{k}(\boldsymbol{p})=\boldsymbol{p}^{T} \boldsymbol{v}_{k}$, for $1 \leq k \leq K+1$, where $v_{k} \in \mathbb{R}_{+}^{K+1}$ is a vector of interference coupling coefficients with the $K+1^{\text {th }}$ component of each vector being $\sigma^{2}$. For a given power vector $\boldsymbol{p}$, the interference (plus noise) in the system is determined by the $K \times$ $(K+1)$ interference coupling matrix $\boldsymbol{V}=\left[\boldsymbol{v}_{1} ; \ldots ; \boldsymbol{v}_{K}\right]$. Since $e^{x}$ is a log-convex function and the point-wise product of two $\log$-convex functions is log-convex, we have that for any user $k \in \mathcal{K}$

$$
\begin{aligned}
\frac{\boldsymbol{v}_{k} e^{\boldsymbol{s}(\lambda)}}{e^{\boldsymbol{s}_{k}(\lambda)}} & \leq\left(\frac{\boldsymbol{v}_{k} e^{\boldsymbol{s}^{(1)}}}{e^{\boldsymbol{s}_{k}^{(1)}}}\right)^{1-\lambda}\left(\frac{\boldsymbol{v}_{k} e^{\boldsymbol{s}^{(2)}}}{e^{s_{k}^{(2)}}}\right)^{\lambda} \\
& =\exp \left((1-\lambda) \log \left(\frac{\boldsymbol{v}_{k} e^{\boldsymbol{s}^{(1)}}}{e^{s_{k}^{(1)}}}\right)+\lambda \log \left(\frac{\boldsymbol{v}_{k} e^{\boldsymbol{s}^{(2)}}}{e^{s_{k}^{(2)}}}\right)\right), \\
& \leq(1-\lambda) \exp \log \left(\frac{\boldsymbol{v}_{k} e^{\boldsymbol{s}^{(1)}}}{e^{s_{k}^{(1)}}}\right)+\lambda \exp \log \left(\frac{\boldsymbol{v}_{k} e^{\boldsymbol{s}^{(2)}}}{e^{s_{k}^{(2)}}}\right) \\
& =(1-\lambda)\left(\frac{\boldsymbol{v}_{k} e^{\boldsymbol{s}^{(1)}}}{e^{\boldsymbol{s}_{k}^{(1)}}}\right)+\lambda\left(\frac{\boldsymbol{v}_{k} e^{\boldsymbol{s}^{(2)}}}{e^{s_{k}^{(2)}}}\right) .
\end{aligned}
$$

We have shown the joint convexity of the function $\mathcal{I}_{k}(\psi(s)) / \psi\left(s_{k}\right)$ with respect to $\boldsymbol{s}$. Hence, Requirement 2 is satisfied.

Now to show, that Requirement 1 is satisfied. We have to consider the feasible SINR region without power con- 
straints. Hence, we can analyze the SIR region instead of the SINR region. Let $v_{k} \in \mathbb{R}_{+}^{K}$ is a vector of interference coupling coefficients without noise and the interference in the system is determined by the $K \times K$ interference coupling matrix $\boldsymbol{V}=\left[\boldsymbol{v}_{1} ; \ldots ; \boldsymbol{v}_{K}\right]$. The feasible SIR region can be written as, $\mathcal{F}=\{\hat{\boldsymbol{\gamma}}>\mathbf{0} \mid \rho(\operatorname{diag}\{\hat{\boldsymbol{\gamma}}\} \boldsymbol{V}) \leq 1\}$, where $\rho(\hat{\gamma}):=\rho(\operatorname{diag}\{\hat{\gamma}\} \boldsymbol{V})$ is the spectral radius of the interference coupling matrix. The spectral radius is log-convex after a change of variable $\hat{\boldsymbol{\gamma}}=e^{\boldsymbol{u}}$, where $\boldsymbol{u}$ is the logarithmic SIR. It is observed, that the SIR set $\mathcal{F}$ is convex on a logarithmic scale. Hence, satisfying Requirement 1 . Then, we have our desired result.

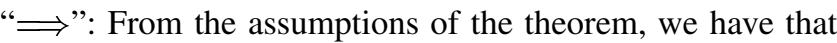
for all linear interference functions, the supportable QoS region (without power control) is convex with respect to $\boldsymbol{s}$, i.e., $\left(\frac{1}{\gamma_{k}}\right)^{\alpha}=\left(\frac{\mathcal{I}_{k}(\psi(\boldsymbol{s}))}{\psi\left(s_{k}\right)}\right)^{\alpha}$, is a convex function with respect to $\boldsymbol{s}=$ $\left[s_{1}, \ldots, s_{K}\right]$. We shall investigate the two-user case, without any loss of generality. Therefore, we check for the convexity of $\left(\frac{\psi\left(s_{2}\right)}{\psi\left(s_{1}\right)}\right)^{\alpha}$, for a certain fixed $\alpha>0$. We fix the power of user 2 . Hence, we fix the value $s_{2}$ and check for the convexity of $1 /\left(\psi\left(s_{1}\right)\right)^{\alpha}$, for all $\alpha>0$. Then, we have that $\left(\psi^{\prime}\left(s_{1}\right)\right)^{2}-$ $\frac{1}{\alpha+1} \psi^{\prime \prime}\left(s_{1}\right) \psi\left(s_{1}\right) \geq 0$. Taking the limit of $\alpha \rightarrow 0$, we obtain

$$
\left(\psi^{\prime}\left(s_{1}\right)\right)^{2}-\psi^{\prime \prime}\left(s_{1}\right) \psi\left(s_{1}\right) \geq 0 .
$$

Since, Requirement 1 is satisfied, $\psi$ is log-convex and

$$
\left(\psi^{\prime}\left(s_{1}\right)\right)^{2}-\psi^{\prime \prime}\left(s_{1}\right) \psi\left(s_{1}\right) \leq 0 .
$$

From (9) and (10) we have that

$$
\left(\psi^{\prime}(s)\right)^{2}-\psi^{\prime \prime}(s) \psi(s)=0 .
$$

If $\psi$ is a solution of (11), with $\psi(s)>0$ for $s \in \mathbf{S}$, then we have that $\left(\psi^{\prime}(s)\right)^{2}-\psi^{\prime \prime}(s) \psi(s) /(\psi(s))^{2}=0$. This gives us $\frac{d}{d s}\left(\frac{\psi^{\prime}(s)}{\psi(s)}\right)=0$, i.e., $\frac{\psi^{\prime}(s)}{\psi(s)}=\mu$. Since, $\psi$ is strictly monotonic increasing (from our assumptions) we have that $\mu>0$. Therefore, $\frac{d \psi}{\psi}=\mu d s$, i.e., $\psi(s)=c \exp (\mu s)$.

Remark 3: Theorem 1 has addressed point 1 of the Problem 1.

We have that $\frac{\mathcal{I}_{k}(\psi(\boldsymbol{s}))}{\psi\left(s_{k}\right)}=\frac{c \mathcal{I}_{k}(\exp (\mu \boldsymbol{s}))}{c \exp \left(\mu s_{k}\right)}=\frac{\mathcal{I}_{k}(\exp (\mu \boldsymbol{s}))}{\exp \left(\mu s_{k}\right)}$. Hence, we can choose $c=1$, The constant $c$ in the statement of Theorem 1 has the role of an initialization in the differential equation in the proof and $c$ has no impact on the SINR.

Historically, there have been a number of different motivations for utilizing the log-scale for measuring power in communication systems, e.g., the logarithmic nature allowing a representation of a very large range of ratios can be represented by a convenient number. Theorem 1, provides another reason as to why it is advantageous to work in the log-domain, instead of the power domain. Theorem 1 has been proved for the case, when we can scale the noise. We now analyze the case, when we have noise and we do not scale the noise.

Theorem 2: Function $\psi$ satisfies Requirements 1 and 2, if and only if there exists $a \mu, c>0$ such that $\psi\left(s_{k}\right)=c \exp \left(\mu s_{k}\right)$, $c>0, s \in \mathbb{R}$, for $k \in \mathcal{K}$.

Proof: "£”: Since, we are analyzing the case, when we do not allow the scaling of noise, let $\underline{\boldsymbol{s}}^{(1)}=\left[\boldsymbol{s}^{(1)}, \sigma^{2}\right]^{T}$ and $\underline{\boldsymbol{s}}^{(2)}=\left[\boldsymbol{s}^{(2)}, \sigma^{2}\right]^{T}$, where $\boldsymbol{s}^{(1)}, \boldsymbol{s}^{(2)} \in \mathbb{R}^{K}$. Let $\underline{\boldsymbol{s}}(\lambda)=(1-$ $\lambda) \underline{\boldsymbol{s}}^{(1)}+\lambda \underline{\boldsymbol{s}}^{(2)}$. Then,

$$
\begin{aligned}
\underline{\boldsymbol{s}}(\lambda) & =(1-\lambda)\left(\boldsymbol{s}^{(1)}, \sigma^{2}\right)+\lambda\left(\boldsymbol{s}^{(2)}, \sigma^{2}\right) \\
& =(1-\lambda) \boldsymbol{s}^{(1)}+\lambda \boldsymbol{s}^{(2)},
\end{aligned}
$$

where $\boldsymbol{s}^{(1)}, \boldsymbol{s}^{(2)} \in \mathbb{R}^{K}$. The rest of the proof follows exactly as the proof of Theorem 1 (the converse direction).

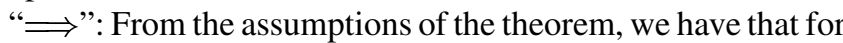
all linear interference functions, $\left(\frac{1}{\gamma_{k}}\right)^{\alpha}=\left(\frac{\mathcal{I}_{k}(\psi(\boldsymbol{s}))}{\psi\left(s_{k}\right)}\right)^{\alpha}$ is jointly convex with respect to $\boldsymbol{s}=\left[s_{1}, \ldots, s_{K}\right]$, for all $\alpha>0$. Now, consider the function

$$
\left(\frac{\mathcal{I}_{k}(\psi(\boldsymbol{s}))}{\psi\left(s_{k}\right)}\right)^{\alpha}=\left(\frac{\sum_{j \in \mathcal{K} \backslash k} v_{k j} \psi\left(s_{j}\right)+v_{k(K+1)} \sigma^{2}}{\psi\left(s_{k}\right)}\right)^{\alpha} .
$$

As $v_{k(K+1)} \rightarrow 0$, the function (13) tends to the noise free case, i.e., $\frac{\sum_{j \in \mathcal{K} \backslash k} v_{k j} \psi\left(s_{j}\right)}{\psi\left(s_{k}\right)}$. The noise free case is identical to the case, when we can scale the noise. Furthermore, we know that the limit function of a sequence of convex functions is convex. Now we can follow the same steps as in the proof of Theorem 1 (the forward direction).

Remark 4: Since, we can apply the same proof technique as in the proof of Theorem 2, w.l.o.g. we prove all theorems throughout the paper with noise scaling.

Remark 5: The composition of a convex and a concave function need not be convex. A function $f$ is convex, if and only if the function $-f$ is concave. Hence, it is important to check for the convexity of a function of inverse SINR.

Theorem 1 presents a result, from the perspective an arbitrary user $k$. We now extend the result to a system level perspective in Theorem 3 below.

Theorem 3: Function $\psi$ satisfies Requirements 1 and 3, if and only if there exists scalars $c, \mu>0$ such that $\psi\left(s_{k}\right)=$ $c \exp \left(\mu s_{k}\right)$, where $s \in \mathbb{R}$, for $1 \leq k \leq K+1$.

Function $\psi$ satisfies Requirements 1 and 3 , if and only if there exists a $\mu>0$ such that $\psi\left(s_{k}\right)=\exp \left(\mu s_{k}\right)$, where $s \in \mathbb{R}$, for all $k \in \mathcal{K}$.

Proof: We know that under Requirement 1, the function $\mathcal{I}_{k}(\psi(\boldsymbol{s})) / \psi\left(s_{k}\right)$ is convex, if and only if $\psi\left(s_{k}\right)=\exp \left(\mu s_{k}\right)$ for $\mu>0$ (from Theorem 1). Therefore, it is sufficient to prove that the function (6), with $\sum_{k \in \mathcal{K}} \omega_{k}=1$ is convex, if and only if $\mathcal{I}_{k}(\psi(\boldsymbol{s})) / \psi\left(s_{k}\right)$ is convex.

" tion. We know that the concatenation of convex functions is convex. Hence, this direction can be easily verified. Hence, we skip the proof.

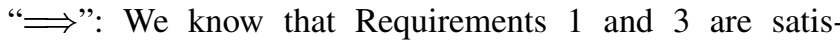
fied. We can choose $g(x)=x^{\alpha}$, for all $\alpha>0$. Then, $\sum_{k \in \mathcal{K}} \omega_{k}\left(\frac{\mathcal{I}_{k}(\psi(\boldsymbol{s}))}{\psi\left(\boldsymbol{s}_{k}\right)}\right)^{\alpha}$ with $\sum_{k \in \mathcal{K}} \omega_{k}=1$ and $\boldsymbol{\omega}>\mathbf{0}$ is jointly convex with respect to $\boldsymbol{s}$, for all $\alpha>0$. Let us choose weight vectors as follows:

$$
\omega_{k}^{(n)}= \begin{cases}1-\frac{1}{n} & k=j \\ \frac{1}{(K-1) n} & k \neq j .\end{cases}
$$


Taking the limit as $n$ tends to $\infty$, we obtain $\left(\frac{\mathcal{I}_{j}(\psi(\boldsymbol{s}))}{\psi\left(\boldsymbol{s}_{j}\right)}\right)^{\alpha}=$ $\lim _{n \rightarrow \infty} \sum_{k \in \mathcal{K}} \omega_{k}^{(n)}\left(\frac{\mathcal{I}_{k}(\psi(\boldsymbol{s}))}{\psi\left(s_{k}\right)}\right)^{\alpha}$. The limit function of a sequence of convex function is convex. Therefore, $\left(\mathcal{I}_{j}(\psi(\boldsymbol{s})) / \psi\left(s_{j}\right)\right)^{\alpha}$ is jointly convex in $\boldsymbol{s}$. Therefore, Requirements 1 and 2 are satisfied. Then, from Theorem 1, we have our desired result.

Remark 6: Theorem 3 has addressed point 1 and point 2 of the Problem 3.

From Theorems 1 and 3, we have an equivalence between Requirements 2 and 3 . We have established that for linear interference functions the unique transformation that satisfies our requirements and allows us to obtain convex optimization problems is the exponential function $\psi(x)=c \exp (\mu x)$, with $c$, $\mu>0$. This $\psi$ will be utilized in Section III-B. Furthermore, we have shown that the exponential function is the unique mapping if we would like the natural and practical requirement (3) to be satisfied. We would now like to extend our intuition to the case beyond the framework of linear interference functions.

\section{B. Analysis of Convexity Properties of Resource Allocation Problems Beyond Linear Interference Functions}

In this section, we shall extend certain results obtained for linear interference functions to a larger class of interference functions. We are interested in finding the largest class of interference functions, which allow us to apply convex optimization techniques to certain non-convex problems. We shall assume that $\psi(x)=c \exp (\mu x)$, with $c, \mu>0$. Then, (6) is a weighted sum of functions of inverse SINR in the $s$ domain. Hence, it plays the role of a loss function in wireless systems. Intuitively, while tackling such a problem we would like to minimize such a function so as to optimize the satisfaction of the users in the system. We now present a result, that clarifies when such a function can be optimized by means of a convex optimization techniques.

Theorem 4: Let $\psi(x)=c \exp (\mu x)$, with $c, \mu>0$. Then, (6) is jointly convex with respect to $\boldsymbol{s} \in \mathbb{R}^{K+1}$ for all weight vectors $\boldsymbol{\omega}>\mathbf{0}$ and for all convex, continuous and increasing functions $g_{1}, \ldots, g_{K}$, if and only if the functions $\mathcal{I}_{k}\left(e^{\boldsymbol{s}}\right) / e^{s_{k}}$ for all $k \in \mathcal{K}$ are jointly convex with respect to $s \in \mathbb{R}^{K+1}$.

Let $\psi(x)=c \exp (\mu x)$, with $c, \mu>0$. Then, the function (6) is jointly convex with respect to $s \in \mathbb{R}^{K}$ for all weight vectors $\boldsymbol{\omega}>\mathbf{0}$ and for all convex, continuous and increasing functions $g_{1}, \ldots, g_{K}$, if and only if the functions $\mathcal{I}_{k}\left(e^{\boldsymbol{s}}, \sigma^{2}\right) / e^{s_{k}}$ for all $k \in \mathcal{K}$ are jointly convex with respect to $s \in \mathbb{R}^{K}$.

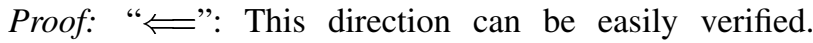
When $g_{k}$ and $\mathcal{I}_{k}\left(e^{\boldsymbol{s}}\right) / e^{s_{k}}$ are convex functions, we know that $g_{k}\left(\mathcal{I}_{k}\left(e^{s}\right) / e^{s_{k}}\right)$ is convex. Furthermore, since the weighted sum of convex functions is convex, we obtain our desired result.

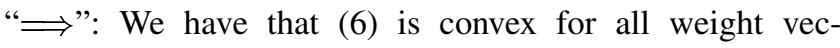
tors $\boldsymbol{\omega}>\mathbf{0}$ and for all convex, continuous and increasing functions $g_{1}, \ldots, g_{K}$. Choose $g_{k}(x)=x$ for all users $k \in \mathcal{K}$. Let us choose weight vectors as in the proof of Theorem 3. Taking the limit as $n \rightarrow \infty$, we have $\mathcal{I}_{j}\left(e^{s}\right) / e^{s_{j}}=\lim _{n \rightarrow \infty} \sum_{k \in \mathcal{K}} \omega_{k}^{(n)} \mathcal{I}_{k}\left(e^{s}\right) / e^{s_{k}}$. The limit function of a sequence of convex function is convex. Therefore, $\mathcal{I}_{j}\left(e^{\boldsymbol{s}}\right) / e^{s_{j}}$ is jointly convex with respect to $\boldsymbol{s}$.
Remark 7: Theorem 4 has addressed point 3 of the Problem 3.

The largest class of interference functions, resulting in convex resource allocation problems would be equal to or larger than log-convex interference functions.

If interference functions $\mathcal{I}_{1}, \ldots, \mathcal{I}_{K}$ are log-convex interference functions, then $\sum_{k \in \mathcal{K}} \omega_{k} \log \frac{e^{s_{k}}}{\mathcal{I}_{k}\left(e^{s}\right)}-\sum_{k \in \mathcal{K}} \omega_{k} \log \frac{\mathcal{I}_{k}\left(e^{s}\right.}{e^{s_{k}}}$ is jointly concave [36].

From Theorems 1 and 3, we know that for all strictly monotonic increasing, continuous and convex functions $g_{k}$ and for all weight vectors $\boldsymbol{\omega}>\mathbf{0}$, it is sufficient to check for the joint convexity of the function $\sum_{k \in \mathcal{K}} \omega_{k} g_{k}\left(\mathcal{I}_{k}\left(e^{\boldsymbol{s}}\right) / e^{s_{k}}\right)$ with respect to $s$. Hence, we define a new class of interference functions below:

Definition 4: $\mathcal{C}$ interference functions: A general interference function $\mathcal{I}_{k}$ is said to a be $\mathcal{C}$ interference function if the function $\mathcal{I}_{k}(\psi(\boldsymbol{s})) / \psi\left(s_{k}\right)$ is jointly convex with respect to $\boldsymbol{s}$, where $\psi\left(s_{k}\right)=e^{\mu s_{k}}$, for $k \in \mathcal{K}$, with $\mu>0$.

The inclusion of the different families of interference functions is as follows: Convex interference functions $\subset$ Log-convex interference functions $\subset \mathcal{C}$ interference functions $\subset$ General interference functions. We now define the following function, which we shall utilize in analyzing the convexity of the function $\mathcal{I}_{k}\left(e^{\boldsymbol{s}}\right) / e^{s_{k}}$.

$$
\tilde{f}_{\mathcal{I}_{k}}(\boldsymbol{\omega}):=\inf _{\underline{\boldsymbol{p}}>\mathbf{0}} \frac{\exp \left(\frac{\mathcal{I}_{k}(\underline{p})}{p_{k}}\right)}{\prod_{l=1}^{K}\left(p_{l}\right)^{\omega_{l}}}
$$

The function $\tilde{f}_{\mathcal{I}_{k}}$ defined by (14) is log-concave, for all users $k \in \mathcal{K}$. A function $f: \mathbb{R}^{K+1} \mapsto \mathbb{R}_{+}$is log-concave on $\mathbb{R}^{K+1}$, if and only if $\log f$ is concave or equivalently $f(\boldsymbol{s}(\lambda)) \geq f\left(\boldsymbol{s}^{(1)}\right)^{1-\lambda} f\left(\boldsymbol{s}^{(2)}\right)^{\lambda}$, for all $\lambda \in(0,1)$, $\boldsymbol{s}^{(1)}, \boldsymbol{s}^{(2)} \in \mathbb{R}^{K+1}$, where $\boldsymbol{s}(\lambda)=(1-\lambda) \boldsymbol{s}^{(1)}+\lambda \boldsymbol{s}^{(2)}$, $\lambda \in(0,1)$.

Lemma 1: Let the function $\tilde{f}_{\mathcal{I}_{k}}(\boldsymbol{\omega})>0$, where $\tilde{f}_{\mathcal{I}_{k}}(\boldsymbol{\omega})$ is defined in (14). Then, the sum of the weight vectors $\sum_{l \in \mathcal{K}} \omega_{l}=$ 0 .

The proof of Lemma 1 can be found in [37]. From [37], we have the following remarks, which along with Theorem 4 help us obtain a complete characterization of the convexity properties of resource allocation problems for interference coupled systems beyond the case of linear interference functions. These remarks along with Theorem 4 characterize the largest class of interference functions ( $\mathcal{C}$ interference functions), which allow a problem in interference coupled wireless systems to be formulated as a convex optimization problem.

Remark 8: For all $s \in \mathbb{R}^{K+1}$ the function $g_{k}(\boldsymbol{s}):=$ $\mathcal{I}_{k}\left(e^{\boldsymbol{s}}\right) / e^{s_{k}}$ is convex, if and only if

$$
\begin{aligned}
\mathcal{I}_{k}\left(e^{\boldsymbol{s}}\right) & =e^{s_{k}} \log h_{\mathcal{I}_{k}}(\boldsymbol{s}), \quad k \in \mathcal{K}, \text { where } \\
h_{\mathcal{I}_{k}}(\boldsymbol{s}) & :=\sup _{\boldsymbol{\omega}: \tilde{f}_{\mathcal{I}_{k}}(\boldsymbol{\omega})>0} \tilde{f}_{\mathcal{I}_{k}}(\boldsymbol{\omega}) \prod_{l \in \mathcal{K}}\left(e^{s_{l} \omega_{l}}\right) .
\end{aligned}
$$

For all $s \in \mathbb{R}^{K}$ the function $g_{k}(\boldsymbol{s}):=\mathcal{I}_{k}\left(e^{\boldsymbol{s}}, \sigma^{2}\right) / e^{s_{k}}$ is convex, if and only if

$$
\begin{aligned}
\mathcal{I}_{k}\left(e^{\boldsymbol{s}}, \sigma^{2}\right) & =e^{\boldsymbol{s}_{k}} \log h_{\mathcal{I}_{k}}(\boldsymbol{s}), \quad k \in \mathcal{K}, \text { where } \\
h_{\mathcal{I}_{k}}(\boldsymbol{s}) & :=\sup _{\boldsymbol{\omega}: \tilde{f}_{\mathcal{I}_{k}}(\boldsymbol{\omega})>0} \tilde{f}_{\mathcal{I}_{k}}(\boldsymbol{\omega}) \prod_{l \in \mathcal{K}}\left(e^{s_{l} \omega_{l}}\right) .
\end{aligned}
$$


It can be seen in Lemma 1, that the weight vectors $\boldsymbol{\omega}$ can be less than zero. Although this might seem surprising at first glance, it is justified from the fact, that we are now concerned with the optimization of function $\tilde{f}_{\mathcal{I}}(\boldsymbol{\omega})$, which has the SINR as an inverse argument. We are investigating the case in the $\boldsymbol{s}$-domain $\left(\underline{p}=e^{\boldsymbol{s}}\right)$. Hence, a more negative weight implies a larger SINR in the power domain.

We notice in Remark 8, that $\mathcal{I}_{k}\left(e^{\boldsymbol{s}}\right)$ is convex, which is stronger than the condition that it is log-convex, since $\log h_{\mathcal{I}_{k}}(\boldsymbol{s})$ is convex. We see that $\log$-convexity plays a significant role in the analysis. Remark 8 has characterized the class of interference functions $\mathcal{C}$, which leads to the inverse SINR function $\mathcal{I}_{k}\left(e^{\boldsymbol{s}}\right) / e^{s_{k}}$ to be convex in the $\boldsymbol{s}$-domain, for all users $k \in \mathcal{K} . \tilde{f}_{\mathcal{I}}$ has been introduced for the purpose of investigating the convexity properties of SINR.

From Theorem 4 and Remark 8, we make the following observation: The function (6) is jointly convex with respect to $\boldsymbol{s} \in$ $\mathbb{R}^{K+1}$ for all weight vectors $\boldsymbol{\omega}>\mathbf{0}$ and for all convex, continuous and increasing functions $g_{1}, \ldots, g_{K}$, if and only if the functions $\mathcal{I}_{k}\left(e^{\boldsymbol{s}}\right) / e^{s_{k}}$ for all $k \in \mathcal{K}$ possess the structure defined by (15).

Remark 9: Theorem 4 and Remark 8 have together answered point 2, of the Problem 1.

If $\mathcal{I}\left(e^{\boldsymbol{s}}\right)$ is a log-convex interference function, then the corresponding function $\log \left(\gamma\left(e^{\boldsymbol{s}}\right)\right)$ is convex, i.e., $\gamma\left(e^{\boldsymbol{s}}\right) \leq\left(\gamma^{(1)}\right)^{1-\lambda}\left(\gamma^{(2)}\right)^{\lambda}$, where $\gamma\left(e^{\boldsymbol{s}^{(1)}}\right)=\gamma^{(1)}$ and $\gamma\left(e^{\boldsymbol{s}^{(2)}}\right)=\gamma^{(2)}$. If $\mathcal{I}\left(e^{\boldsymbol{s}}\right)$ is a $\mathcal{C}$ interference function, then we have that the corresponding function $\gamma\left(e^{\boldsymbol{s}}\right)$ is convex. Let $\mathcal{I}^{(n)}, n \in \mathbb{N}$ be a convergent sequence on log-convex interference functions and $\lim _{n \rightarrow \infty} \mathcal{I}^{(n)}=\mathcal{I}^{*}$. Then, $\mathcal{I}^{*}$ is also a log-convex interference function. Hence, we have that the class of log-convex interference functions is closed with respect to point wise convergence. However, they (the class of log-convex interference functions) are not dense with respect to the class of $\mathcal{C}$ interference functions.

The class of $\mathcal{C}$ interference functions is much larger than the class of log-convex interference functions. These results extend the class of interference coupled systems to which convex optimization techniques can be successfully applied. The class of $\mathcal{C}$ interference functions is the largest class of interference functions, permitting the use of convex optimization techniques to solve certain non-convex problems.

Example 4: Consider the function $q_{\alpha}(x)=x$, for $\alpha \geq 1$. We are interested in minimizing the function $\sum_{k \in \mathcal{K}} \omega_{k}\left(\frac{\mathcal{I}_{k}\left(e^{s}\right)}{e^{s_{k}}}\right)^{\alpha_{k}}$, with $\alpha_{k} \geq 1$, for all users $k \in \mathcal{K}$. Such a problem is met in the form of minimizing the weighted probability of errors. Here, the probability of error for user $k, k \in \mathcal{K}$ with diversity order $\alpha_{k}$ for user $k$ can be approximated as $1 /\left(e^{s_{k}} / \mathcal{I}_{k}\left(e^{\boldsymbol{s}}\right)\right)^{\alpha_{k}}$. We see an example of such a function in [38]. A strategy for system resources by joint optimization of transmit powers and beamformers for minimizing the sum of weighted inverse SIR was considered. In [35], a method for choosing weighting factors so that the sum optimization approach achieves optimal max-min fairness was provided.

We now investigate the possibility of obtaining a similar characterization for the concave case in Section IV.

\section{ANALYSIS OF RESOURCE ALlOCATION PROBLEMS: CONCAVE CASE}

In this section, we check for a transformation of the problem from the power domain to the $\boldsymbol{s}$-domain, with the hope that the resulting function is jointly concave with respect to $\boldsymbol{s}$. The feasible SINR region is convex on the logarithmic scale (similar to the convex case displayed in Fig. 1, we can have a figure for the concave case). This does not imply that $\log \frac{p_{1}}{v_{11} p_{1}+v_{12} p_{2}+\sigma^{2}}$ is jointly concave, nor that

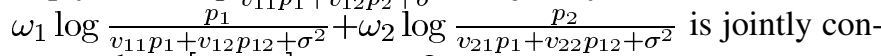
cave, where $\left[\omega_{1}, \omega_{2}\right]=\boldsymbol{\omega} \geq \mathbf{0}$.

\section{A. Analysis of Concavity Properties of Resource Allocation Problems for Linear Interference Functions}

We now present an impossibility result (Theorem 5), which has implications on the concavity properties of resource allocation strategies. These resource allocation strategies aim to maximize functions of SINR in interference coupled wireless systems. We recollect that $\psi(s)=e^{\mu s}, \mu>0$ always leads to jointly convex behavior with respect to $s$, for all linear interference functions (Theorem 1).

Theorem 5: There exists no transformation $\psi$, such that for all linear interference functions, the function $\psi\left(s_{k}\right) / \mathcal{I}_{k}(\psi(\boldsymbol{s}))$, for $1 \leq k \leq K+1$ is jointly concave with respect to $\boldsymbol{s}$.

Proof: For the sake of obtaining a contradiction, assume that the statement of Theorem 5 is not true, i.e., there exists such a function. Choose $\mathcal{I}_{k}(\underline{\boldsymbol{p}})=\sum_{j \in \mathcal{K} \backslash k} v_{j} p_{j}$ and fix $s_{j}$, for all $1 \leq j \leq K+1$ and $j \neq k$. Then, the function $\psi\left(s_{k}\right) / \sum_{j \in \mathcal{K} \backslash k} v_{j} \psi\left(s_{j}\right)$ is concave with respect to $s_{k}$, i.e., the transformation $\psi$ is itself concave.

Now fix $s_{1}$ (w.l.o.g) and consider the following expression $\psi\left(s_{1}\right) / \psi\left(s_{2}\right)$, with $s_{2} \in \mathbf{S}$. This implies, that $1 / \psi\left(s_{2}\right)$ is a concave function. Now, choose $s_{2}^{(1)}, s_{2}^{(2)} \in \mathbf{S}$, such that $s_{2}^{(1)} \neq s_{2}^{(2)}$ arbitrarily. We have that $\psi\left(s_{2}^{(1)}\right) \neq \psi\left(s_{2}^{(1)}\right)$. Let $s_{2}(\lambda):=$ $(1-\lambda) s_{2}^{(1)}+\lambda s_{2}^{(2)}$. Then, we have

$$
\frac{1}{\psi\left(s_{2}(\lambda)\right)} \geq(1-\lambda) \frac{1}{\psi\left(s_{2}^{(1)}\right)}+\lambda \frac{1}{\psi\left(s_{2}^{(2)}\right)} \text {. }
$$

On the other hand, we have $\psi\left(s_{2}(\lambda)\right) \geq(1-\lambda) \psi\left(s_{2}^{(1)}\right)+$ $\lambda \psi\left(s_{2}^{(2)}\right)$ (since $\psi$ is concave). This gives us the following expression:

$$
\begin{aligned}
\frac{1}{\psi\left(s_{2}(\lambda)\right)} & \leq \frac{1}{(1-\lambda) \psi\left(s_{2}^{(1)}\right)+\lambda \psi\left(s_{2}^{(2)}\right)} \\
& <(1-\lambda) \frac{1}{\psi\left(s_{2}^{(1)}\right)}+\lambda \frac{1}{\psi\left(s_{2}^{(2)}\right)} .
\end{aligned}
$$

The strict inequality (above) follows from the fact that the function $1 / x$ is strictly convex and we have our required contradiction with (16).

We have proved the statement of Theorem 5 for the case, when we can scale the noise. Similarly, we can easily prove Theorem 5 for the noise free case.

Theorem 6: There exists no transformation $\psi$, such that for all linear interference functions, the function 
$\psi\left(s_{k}\right) / \mathcal{I}_{k}\left(\psi(\boldsymbol{s}), \sigma^{2}\right)$, for all $1 \in \mathcal{K}$ is jointly concave with respect to $s$.

Proof: The proof follows the same direction as the proof of Theorem 2 and Theorem 5 . We consider the function

$$
\frac{\psi\left(s_{k}\right)}{\sum_{j \in \mathcal{K} \backslash k} v_{k j} \psi\left(s_{j}\right)+v_{k(K+1)} \sigma^{2}} .
$$

As $v_{k(K+1)} \rightarrow 0$, we have that (17) tends to the noise free case, which is the same as the case with noise scaling (Theorem 2). Furthermore, we know that the limit function of a sequence of concave functions is concave. Since, here we have that the limit function is not concave, we can conclude that there exist individual sequences, which are not concave either. Hence, we have our desired result.

We have observed, that the concavity of $g$, e.g. $g(x)=x$ is not sufficient to ensure the joint concavity of $g\left(\psi\left(s_{k}\right) / \mathcal{I}_{k}(\psi(\boldsymbol{s}))\right), k \in \mathcal{K}$ with respect to $\boldsymbol{s}$ for a certain transformation. Hence, we need to restrict the utility functions $g$, such that we can further investigate the joint concavity of our desired function $\psi\left(s_{k}\right) / \mathcal{I}_{k}(\psi(\boldsymbol{s}))$. The necessary condition, which ensures joint concavity will be presented in Lemma 2 below.

Lemma 2: Let a strictly monotonic increasing and twice continuously differentiable function $\psi$ satisfy Requirement 1 . Let $g$ be a monotonic increasing function. Let $g\left(\psi\left(s_{k}\right) / \mathcal{I}_{k}(\psi(\boldsymbol{s}))\right)$ for $1 \leq k \leq K+1$ be jointly concave with respect to $\boldsymbol{s}$ for all linear interference functions $\mathcal{I}_{1}, \ldots, \mathcal{I}_{K}$. Then, $g\left(e^{x}\right)$ is concave.

Let a strictly monotonic increasing and twice continuously differentiable function $\psi$ satisfy Requirement 1 . Let $g$ be a monotonic increasing function. Let $g\left(\psi\left(s_{k}\right) / \mathcal{I}_{k}\left(\psi(\boldsymbol{s}), \sigma^{2}\right)\right)$ for all $k \in \mathcal{K}$ be jointly concave with respect to $\boldsymbol{s}$ for all linear interference functions $\mathcal{I}_{1}, \ldots, \mathcal{I}_{K}$. Then, $g\left(e^{x}\right)$ is concave.

Proof: Choose $x_{1}, x_{2} \in \mathbb{R}$ arbitrarily. Then, for $x(\lambda)=(1-\lambda) x_{1}+\lambda x_{2}$ and from the log-convexity of the function $\psi$, we obtain the following inequality: $\psi(x(\lambda)) \leq$ $\left(\psi\left(x_{1}\right)\right)^{1-\lambda}\left(\psi\left(x_{2}\right)\right)^{\lambda}$. Since, $g$ is a monotonic increasing function, we have that $g(\psi(x(\lambda))) \leq g\left(\left(\psi\left(x_{1}\right)\right)^{1-\lambda}\left(\psi\left(x_{2}\right)\right)^{\lambda}\right)$. Furthermore, from the concavity of $g(\psi(x))$, we have that $(1-\lambda) g\left(\psi\left(x_{1}\right)\right)+\lambda g\left(\psi\left(x_{2}\right)\right) \leq g\left(\psi\left(x_{1}\right)^{1-\lambda} \psi\left(x_{2}\right)^{\lambda}\right)$. Now, let $y_{1}, y_{2} \in \mathbb{R}$ be arbitrarily chosen. We choose $x_{1}, x_{2}$ such that $\psi\left(x_{k}\right)=e^{y_{k}}$, for $k \in\{1,2\}$. This is possible due to our assumption on the function $\psi$. Let $y(\lambda)=(1-\lambda) y_{1}+\lambda y_{2}$. Then, we have that $(1-\lambda) g\left(e^{y_{1}}\right)+\lambda g\left(e^{y_{2}}\right) \leq g\left(e^{y(\lambda)}\right)$, i.e., $g\left(e^{y}\right)$ is concave.

It can be seen that $0 \geq\left(g\left(e^{y}\right)\right)^{\prime \prime}=\left(g^{\prime}\left(e^{y}\right) e^{y}\right)^{\prime}=$ $g^{\prime \prime}\left(e^{y}\right) e^{2 y}+g^{\prime}\left(e^{y}\right) e^{y}$. We have observed that the concavity of $g\left(e^{x}\right)$ is a necessary condition to ensure the joint concavity of the function $g\left(\psi\left(s_{k}\right) / \mathcal{I}_{k}(\psi(\boldsymbol{s}))\right)$ with respect to $\boldsymbol{s}$, for all $k \in \mathcal{K}$.

We have seen in Lemma 2, the existence of a function $\psi$ such that the function $g\left(\psi\left(s_{k}\right) / \mathcal{I}_{k}(\psi(\boldsymbol{s}))\right)$, for all $k \in \mathcal{K}$ is jointly concave with respect to $\boldsymbol{s}$, for all functions $g \in \mathcal{E C}$ onc. We now show in Theorem 7, that the function $\psi=c_{1} \exp (\mu s)$, is up to two constants $c_{1}, \mu$ the unique transformation, which ensures the joint concavity of $g\left(\psi\left(s_{k}\right) / \mathcal{I}_{k}(\psi(\boldsymbol{s}))\right)$, for all linear interference functions and for all utility functions $g \in \mathcal{E C}$ onc. We briefly compare this situation with the convex case, i.e., minimizing the function $g\left(\mathcal{I}_{k}(\boldsymbol{s}) / \psi\left(s_{k}\right)\right)$, where $\psi$ is the exponential function. For the case of linear interference functions and for all strictly monotonic increasing, continuous and convex functions $g$, we have that $g\left(\mathcal{I}_{k}(\boldsymbol{s})\right) / \psi\left(s_{k}\right)$ is jointly convex with respect to $s$. In the convex case we did not require any further restrictions.

Theorem 7: Let a strictly monotonic increasing and twice continuously differentiable function $\psi$ satisfy Requirement 1 . The function $g_{k}\left(\psi\left(s_{k}\right) / \mathcal{I}_{k}(\psi(\boldsymbol{s}))\right)$ is jointly concave with respect to $\boldsymbol{s} \in \mathbb{R}^{K+1}$ for all linear interference functions $\mathcal{I}_{1}, \ldots, \mathcal{I}_{K}$ and for all $g_{k} \in \mathcal{E C}$ onc, if and only if $\psi(s)=c_{1} \exp (\mu s)$, with $c_{1}, \mu>0$.

For all linear interference functions $\mathcal{I}_{1}, \ldots, \mathcal{I}_{K}$ and for all $g \in \mathcal{E C o n c}, g\left(\psi\left(s_{k}\right) / \mathcal{I}_{k}\left(\psi(\boldsymbol{s}), \sigma^{2}\right)\right)$ is jointly concave with respect to $\boldsymbol{s} \in \mathbb{R}^{K}$, if and only if $\psi(s)=c_{1} \exp (\mu s)$, with $c_{1}, \mu>0$.

Proof: “£”: Consider $g_{k}\left(\psi\left(s_{k}\right) / \mathcal{I}_{k}(\psi(\boldsymbol{s}))\right)$ such that $\psi(s)=c_{1} \exp (\mu s)$, with $c_{1}, \mu>0$. Let $\mathcal{I}_{k}$ be a linear interference function for all users $k \in \mathcal{K}$. It can be easily verified that $g\left(e^{\boldsymbol{\mu} s_{k}} / \mathcal{I}_{k}\left(e^{\boldsymbol{\mu} \boldsymbol{s}}\right)\right)$ is jointly concave with respect to $\boldsymbol{s} \in \mathbb{R}^{K+1}$. Hence, we skip the proof.

“””: Let $g_{k}(x)=\log x$. Then, $g_{k}\left(e^{x}\right)$ is in the class $\mathcal{E C}$ onc. Now, let $g_{k} \circ \psi\left(s_{k}\right)=\log \psi\left(s_{k}\right)$. Hence, $g_{k} \circ \psi$ is concave. Furthermore, the function $\psi$ is log-convex, i.e., $g_{k} \circ \psi$ is also convex. Therefore, we choose $s(\lambda)=(1-\lambda) s_{1}+\lambda s_{2}$ and choose $s_{1}=0, s_{2}=1$. Then, we have that

$$
\begin{aligned}
\log \psi(s(\lambda)) & =\log \psi(\lambda) \\
& =(1-\lambda) \log \psi(0)+\lambda \log \psi(1) \\
& =\log \psi(0)+\lambda\left(\log (\underbrace{\frac{\psi(1)}{\psi(0)}}_{>0})\right) .
\end{aligned}
$$

Let $\psi(1) / \psi(0)=\mu$. Then, we have $\psi(\lambda)=\psi(0) e^{\lambda \mu}$, where $\mu>0$.

Remark 10: Theorem 5 and Theorem 7 has addressed point 1 and point 2 of the Problem 2.

We now extend our insight obtained from Theorem 5 and the Lemma 2, beyond the case of linear interference functions.

\section{B. Analysis of Concavity Properties of Resource Allocation Problems Beyond Linear Interference Functions}

In this section, we shall analyze the concavity properties of resource allocation problems for interference functions, beyond the class of linear interference functions. We shall be particularly interested in investigating Problem 4 . It has been established in Section IV-A, that $\psi(x)=c \exp (\mu x)$, with $c, \mu>0$ satisfies our requirements. Now, we check for the joint concavity of $\sum_{k \in \mathcal{K}} \omega_{k} g_{k}\left(\psi\left(s_{k}\right) / \mathcal{I}_{k}(\psi(\boldsymbol{s}))\right)$ for all weight vectors $\boldsymbol{\omega}>\mathbf{0}$ and for all utility functions $g_{k} \in \mathcal{E C}$ onc for the largest possible class of interference functions. Then, we have the following result.

Theorem 8: Let $\psi(x)=c \exp (\mu x)$, with $c, \mu>0$. The function (7) is jointly concave with respect to $s \in \mathbb{R}^{K+1}$ for 
all $\boldsymbol{\omega}>\mathbf{0}$ and for all $g_{k} \in \mathcal{E C}$ onc, if and only if $\mathcal{I}_{1}, \ldots, \mathcal{I}_{K}$ are all log-convex interference functions.

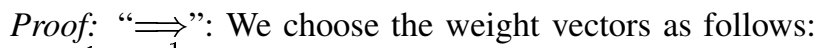
$\omega_{k}^{(n)}=\left\{\begin{array}{ll}1-\frac{1}{n} & k=j \\ \frac{1}{(K-1)} n & k \neq j\end{array}\right.$ Taking the limit as $n \rightarrow \infty$, we have that $e^{\boldsymbol{s}_{j}} / \mathcal{I}_{j}\left(e^{\boldsymbol{s}}\right)=\lim _{n \rightarrow \infty} \sum_{k \in \mathcal{K}} \omega_{k}^{(n)} e^{s_{k}} / \mathcal{I}_{k}\left(e^{\boldsymbol{s}}\right)$. We achieve that $g_{k}\left(e^{s_{k}} / \mathcal{I}_{k}\left(e^{\boldsymbol{s}}\right)\right):=G(\boldsymbol{s})$ is jointly concave with respect to $s$, for all $g \in \mathcal{E} \mathcal{C}$ onc. Since, the limit function of a sequence of concave function is concave. Choose $g_{k}(x)=\log (x)$. Then, for $g \in \mathcal{E C}$ onc we have $\log \mathcal{I}_{k}\left(e^{s}\right)=s+\log G(\boldsymbol{s})$, i.e., $\mathcal{I}_{k}\left(e^{\boldsymbol{s}}\right)$ is log-convex.

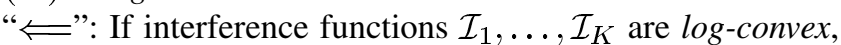
then for arbitrarily chosen $s^{(1)}, s^{(2)}$ and $s(\lambda):=(1-\lambda) s^{(1)}+$ $\lambda s^{(2)}$, we have that

$$
\begin{aligned}
\mathcal{I}_{k}\left(e^{s(\lambda)}\right) & \leq\left(\mathcal{I}_{k}\left(e^{s^{(1)}}\right)\right)^{1-\lambda}\left(\mathcal{I}_{k}\left(e^{s^{(2)}}\right)\right)^{\lambda} \\
\text { i.e., } \quad \frac{e^{s_{k}(\lambda)}}{\mathcal{I}_{k}\left(e^{s(\lambda)}\right)} & \geq\left(\frac{e^{s_{k}^{(1)}}}{\mathcal{I}_{k}\left(e^{s^{(1)}}\right)}\right)^{1-\lambda}\left(\frac{e^{s_{k}^{(2)}}}{\mathcal{I}_{k}\left(e^{s^{(2)}}\right)}\right)^{\lambda} .
\end{aligned}
$$

Then, for a fixed $g_{k} \in \mathcal{E C}$ onc, we have

$$
\begin{aligned}
g_{k}\left(\frac{e^{s_{k}(\lambda)}}{\mathcal{I}_{k}\left(e^{s(\lambda)}\right)}\right) \geq & g_{k}\left(\left(\frac{e^{s_{k}^{(1)}}}{\mathcal{I}_{k}\left(e^{s^{(1)}}\right)}\right)^{1-\lambda}\left(\frac{e^{s_{k}^{(2)}}}{\mathcal{I}_{k}\left(e^{s^{(2)}}\right)}\right)^{\lambda}\right) \\
\geq & (1-\lambda) g_{k}\left(\frac{e^{s_{k}^{(1)}}}{\mathcal{I}_{k}\left(e^{s^{(1)}}\right)}\right) \\
& +\lambda g_{k}\left(\frac{e^{s_{k}^{(2)}}}{\mathcal{I}_{k}\left(e^{s^{(2)}}\right)}\right) .
\end{aligned}
$$

Inequality in (19) follows from the concavity of $g_{k}\left(e^{y}\right)$. Hence, we have that $g_{k}\left(e^{s_{k}} / \mathcal{I}_{k}\left(e^{\boldsymbol{s}}\right)\right)$, for all $k \in \mathcal{K}$ is jointly concave with respect to $\boldsymbol{s}$. Hence, we have that $\sum_{k \in \mathcal{K}} \omega_{k} g_{k}\left(e^{s_{k}} / \mathcal{I}_{k}\left(e^{\boldsymbol{s}}\right)\right)$ for all weight vectors $\boldsymbol{\omega}>\mathbf{0}$ and for all $g_{k} \in \mathcal{E C}$ onc is jointly concave with respect to $\boldsymbol{s}$.

Remark 11: Theorem 8 has completely addressed the Problem 4.

Theorem 9: Let $\psi(x)=c \exp (\mu x)$, with $c, \mu>0$. The function $\left.\sum_{k \in \mathcal{K}} \omega_{k} g_{k}\left(e^{s_{k}} / \mathcal{I}_{k}\left(e^{\boldsymbol{s}}\right), \sigma^{2}\right)\right)$ is jointly concave with respect to $\boldsymbol{s}$ for all $\boldsymbol{\omega}>\mathbf{0}$ and for all $g_{k} \in \mathcal{E C}$ Conc, if and only if $\mathcal{I}_{1}, \ldots, \mathcal{I}_{K}$ are log-convex interference functions.

Proof: " $\Longleftarrow ":$ If interference functions $\mathcal{I}_{k}\left(e^{s_{1}}, \ldots, e^{s_{K}}, \sigma^{2}\right)$, for all $k \in \mathcal{K}$ are log-convex with respect to $s \in \mathbb{R}^{K}$, then with a similar arguments as in the proof of Theorem 6, we have the concavity of $\left.\sum_{k \in \mathcal{K}} \omega_{k} g_{k}\left(e^{s_{k}} / \mathcal{I}_{k}\left(e^{\boldsymbol{s}}\right), \sigma^{2}\right)\right)$, for all $g_{k} \in \mathcal{E C}$ onc.

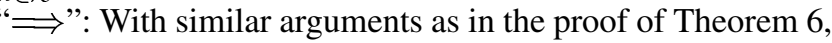
we can prove that $\mathcal{I}_{k}\left(e^{\boldsymbol{s}}, \sigma^{2}\right)$ is log-convex with respect to $\boldsymbol{s} \in$ $\mathbb{R}^{K}$. Then from Theorem 8 in [7], we have that $\mathcal{I}_{k}\left(e^{\boldsymbol{s}}, e^{s_{K+1}}\right)$ is also jointly log-convex.

We contrast the result obtained from Theorem 8 to the convex case. In the convex case, i.e., minimization of (6), where $g_{k}$ is a strictly monotonic increasing, continuous and convex function, it can be observed that with log-convex interference functions (have been discussed in [39]) the function $\sum_{k \in \mathcal{K}} \omega_{k} g_{k}\left(\mathcal{I}_{k}\left(e^{\boldsymbol{s}}\right) / e_{k}^{s}\right)$ is jointly convex with respect to $\boldsymbol{s}$. We shall investigate the possibility of obtaining a larger class of utility functions, which preserves convexity properties of functions of inverse SINR for interference functions, which are not log-convex in Section V.

\section{LARGER ClASS OF UTILITY FUNCTIONS}

We have seen from Theorem 8 and from [36], that log-convex interference functions play a special role in the characterization of concavity properties of resource allocation problems. In [36] the main focus was clarifying the importance of log-convex interference functions. In our paper, we attempt to obtain the largest class of utility functions such that the considered resource allocation problem still possesses convexity properties. We explore the trade-off between the generality of the class of utility functions and the generality of the class of interference functions. We now return to the convex case and present the following result.

Lemma 3: Let $\psi(x)=c \exp (\mu x)$, with $c, \mu>0$. Function (8) is jointly convex with respect to $s \in \mathbb{R}_{+}^{K+1}$ for all linear interference functions, if and only if the function $g\left(e^{x}\right)$ is convex.

Let $\psi(x)=c \exp (\mu x)$, with $c, \mu>0$. Function (8) is jointly convex with respect to $s \in \mathbb{R}_{+}^{K}$ for all linear interference functions, if and only if $g\left(e^{x}\right)$ is convex.

Proof: " $\Longrightarrow$ ": Let $s_{1}=0$. Then $\mathcal{I}_{k}\left(e^{\boldsymbol{s}}\right)=e^{s_{2}}$. Then, for $s_{2}^{(1)}, s_{2}^{(2)}$ arbitrarily chosen and $s_{2}(\lambda)=(1-\lambda) s_{2}^{(1)}+\lambda s_{2}^{(2)}$ we obtain $g\left(e^{s(\lambda)}\right) \leq(1-\lambda) g\left(e^{s_{2}^{(1)}}\right)+\lambda g\left(e^{s_{2}^{(2)}}\right)$, i.e., the function $g\left(e^{x}\right)$ is convex.

" $\Longleftarrow$ ": We have for all linear interference functions $\mathcal{I}$ and $\boldsymbol{s}^{(1)}, \boldsymbol{s}^{(2)}$ arbitrarily chosen and $\boldsymbol{s}(\lambda)=(1-\lambda) \boldsymbol{s}^{(1)}+\lambda \boldsymbol{s}^{(2)}$ that $\mathcal{I}\left(e^{\boldsymbol{s}(\lambda)}\right) \leq\left(\mathcal{I}\left(e^{\boldsymbol{s}^{(1)}}\right)\right)^{1-\lambda}\left(\mathcal{I}\left(e^{\boldsymbol{s}^{(2)}}\right)\right)^{\lambda}$. This give us, that

$$
\begin{aligned}
g\left(\frac{\mathcal{I}\left(e^{\boldsymbol{s}(\lambda)}\right)}{e^{s_{1}(\lambda)}}\right) \leq & g\left(\left(\frac{\mathcal{I}\left(e^{\boldsymbol{s}^{(1)}}\right)}{e^{s_{1}^{(1)}}}\right)^{1-\lambda}\left(\frac{\mathcal{I}\left(e^{\boldsymbol{s}^{(2)}}\right)}{e^{s_{1}^{(2)}}}\right)^{\lambda}\right) \\
\leq & (1-\lambda) g\left(\frac{\mathcal{I}\left(e^{\boldsymbol{s}^{(1)}}\right)}{e^{s_{1}^{(1)}}}\right) \\
& +\lambda g\left(\frac{\mathcal{I}\left(e^{\boldsymbol{s}^{(2)}}\right)}{e^{s_{2}^{(1)}}}\right)
\end{aligned}
$$

is jointly convex with respect to $\boldsymbol{s}$.

Based on Lemma 3 we are now in a position to answer Problem 5.

Theorem 10: The function $\sum_{k \in \mathcal{K}} \omega_{k} g_{k}\left(\mathcal{I}_{k}\left(e^{\boldsymbol{s}}\right) / e^{s_{k}}\right)$ is jointly convex with respect to $s \in \mathbb{R}^{K+1}$ for all monotonic increasing and continuous functions $g_{k}, k \in \mathcal{K}$, for $\boldsymbol{\omega}>\mathbf{0}$ and for all linear interference functions, if and only if $g\left(e^{x}\right)$ is convex.

The function $\sum_{k \in \mathcal{K}} \omega_{k} g_{k}\left(\mathcal{I}_{k}\left(e^{s}, \sigma^{2}\right) / e^{s_{k}}\right)$ is jointly convex with respect to $s \in \mathbb{R}^{K}$ for all monotonic increasing and continuous functions $g_{k}, k \in \mathcal{K}$, for $\boldsymbol{\omega}>\mathbf{0}$ and for all linear interference functions, if and only if $g\left(e^{x}\right)$ is convex. 


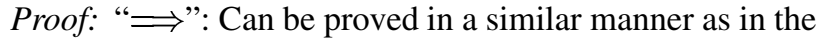
proof of Theorem 7.

"£": This direction follows from Lemma 3 .

Remark 12: Theorem 10 has completely addressed the Problem 5.

We now utilize the additional requirement obtained from Theorem 10 , namely that of the utility functions being in the class $\mathcal{E C o n v}$.

Theorem 11: The function $\sum_{k \in \mathcal{K}} \omega_{k} g_{k}\left(\mathcal{I}_{k}\left(e^{\boldsymbol{s}}\right) / e^{s_{k}}\right)$ is jointly convex with respect to $\boldsymbol{s} \in \mathbb{R}^{K+1}$, for all $\boldsymbol{\omega}>\mathbf{0}$ and for all $g_{k} \in \mathcal{E} \mathcal{C}$ onv with $k \in \mathcal{K}$, if and only if $\mathcal{I}_{1}, \ldots, \mathcal{I}_{K}$ are log-convex interference functions.

The function $\sum_{k \in \mathcal{K}} \omega_{k} g_{k}\left(\mathcal{I}_{k}\left(e^{\boldsymbol{s}}, \sigma^{2}\right) / e^{s_{k}}\right)$ is jointly convex with respect to $\boldsymbol{s} \in \mathbb{R}^{K}$, for all $\boldsymbol{\omega}>\mathbf{0}$ and for all $g_{k} \in \mathcal{E C}$ onv with $k \in \mathcal{K}$, if and only if $\mathcal{I}_{1}, \ldots, \mathcal{I}_{K}$ are log-convex interference functions.

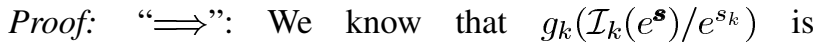
jointly convex for all functions $g_{k} \in \mathcal{E} \mathcal{C}$ onv. We choose, $g_{k}(x)=\log x$, then we have that $g_{k}\left(e^{x}\right)=x$, i.e., $g_{k}^{\prime \prime}\left(e^{x}\right)=0$, i.e., $g_{k}\left(e^{x}\right)$ is convex. This give us that the function $\mathcal{I}_{k}$ is log-convex.

" $\Longleftarrow$ ": Can be proved as in the proof if Theorem 8 .

Remark 13: Theorem 11 has completely addressed the Problem 6 and point 3 of the Problem 3. Hence, Problem 3 has been completely addressed.

Each convex function $g$ has the property that $g\left(e^{x}\right)$ is convex. However, the example that $g(x)=\log x$ shows that even a concave function could be transformed into a convex function. Theorem 11 is very interesting, since we observe that there is a trade-off between the generality of the following two families (classes) of functions:

1) family of utility functions; and

2) family of interference functions.

Theorem 11 has shown that we can obtain convexity for a large class of utility functions, however for a smaller class of interference functions. We have established that

1) $\log$-convex interference functions are the largest class of interference functions, such that the weighted sum of functions $g_{k}$ of inverse SINR are jointly convex in the $s$-domain, for all $g_{k} \in \mathcal{E C}$ onv, with $k \in \mathcal{K}$ for all weight vectors $\boldsymbol{\omega}>\mathbf{0}$, and

2) log-convex interference functions are the largest class of interference functions, such that the weighted sum of functions $g_{k}$ of SINR are jointly concave in the $\boldsymbol{s}$-domain, for all $g_{k} \in \mathcal{E C}$ onc, with $k \in \mathcal{K}$ for all weight vectors $\boldsymbol{\omega}>\mathbf{0}$. The inclusion order of the families of utility functions are as follows: convex case: $\mathcal{C}$ onv $\subset \mathcal{E} \mathcal{C}$ onv, and concave case: $\mathcal{E} \mathcal{C}$ onc $\subset$ Conc.

Example 5: Consider the function $g(x)=x /(1+x)$ (with domain $[0, \infty)$ ), which is concave function. Then, the corresponding function $g\left(e^{x}\right)=e^{x} /\left(1+e^{x}\right)$ (with domain $(-\infty, \infty)$ ), is neither concave nor convex. Hence, we can see that the family of utility functions $\mathcal{E C o n c}$ is smaller, than the family of utility functions, which are concave.

\section{CONCLUSION}

This paper has investigated the possibility of obtaining joint convexity or joint concavity of resource allocation problems.
For the convex case, it has been shown that the exponential transformation is the unique transformation resulting in "convexification" of the resource allocation problem (function of inverse SINR) for linear interference functions. However, in the concave case, for linear interference functions there exists no transformation, which achieves joint concavity. The paper has characterized certain requirements, which expect the transformed feasible SINR region, i.e., the feasible QoS region to be a convex set. Under these natural requirements, the paper has characterized the largest class of utility functions and the largest classes of interference functions, respectively, which individually ensure either joint convexity or joint concavity of the resource allocation problem. In general, convex quadratic programs are globally solvable in polynomial time, whereas non-convex quadratic problems are NP-hard, even when the feasible set is a box or a simplex [40]. The paper has elucidated that the largest class of interference functions, which ensure joint concavity for resource allocation strategies are the logconvex interference functions. The paper has extended previous literature on log-convex interference functions and established boundaries on the class of problems in wireless systems, which are jointly convex or jointly concave. Furthermore, it is noteworthy to observe that the interesting paper [41] states the following. For certain examples of objective functions in wireless networks, e.g. weighted sum of utility maximization problems (where utility is a function of SINR and the objective function subject to certain constraints, e.g. individual power constraints) the resulting problem is NP-hard. Now, let us choose utility functions $u_{1}, \ldots, u_{k} \notin \mathcal{E} \mathcal{C}$ onc. Furthermore, let $\mathcal{I}_{1}, \ldots, \mathcal{I}_{k}$ be linear interference functions. Then, there exists examples such that maximizing the function defined in (2) can be transformed into a convex problem. This example lies outside the framework presented in our paper. However, as can be observed it could still be converted into a convex problem. Hence, it would be interesting to better understand the structure of NP-hard problems and in turn invest further thought into understanding the demarcation between the classes of "convexificable" and "non-convexificable" resource allocation problems in interference coupled systems and the possible modifications in interference coupling constellations, which transition a problem from one class to the other.

\section{APPENDIX}

INTERFERENCE FUNCTIONS

Definition 5: Interference functions: We say that $\mathcal{I}$ : $\mathbb{R}_{+}^{K+1} \mapsto \mathbb{R}_{+}$is an interference function if the following axioms are fulfilled:

$A 1$ conditional positivity $\mathcal{I}(\underline{p})>0$ if $\underline{\boldsymbol{p}}>\mathbf{0}$

$A 2$ scale invariance $\mathcal{I}(\alpha \underline{p}) \stackrel{=}{=} \alpha \mathcal{I}(\underline{p}), \bar{\forall} \alpha \in \mathbb{R}_{+}$

$A 3$ monotonicity $\mathcal{I}(\boldsymbol{p}) \geq \mathcal{I}(\hat{\boldsymbol{p}})$ if $\boldsymbol{p} \geq \hat{\boldsymbol{p}}$

$A 4$ strict monotonicity $\mathcal{I}(\underline{\boldsymbol{p}})>\mathcal{I}(\underline{\hat{\boldsymbol{p}}})$ if $\underline{\boldsymbol{p}} \geq \underline{\hat{\boldsymbol{p}}}$,

$$
p_{K+1}>\hat{p}_{K+1} \text {. }
$$

Note that we require that $\mathcal{I}(\underline{\boldsymbol{p}})$ is strictly monotone with respect to the last component $p_{K+1}$. An example is $\mathcal{I}(\underline{p})=\boldsymbol{v}^{T} \boldsymbol{p}+$ $\sigma^{2}$, where $\boldsymbol{v} \in \mathbb{R}_{+}^{K}$ is a vector of interference coupling coefficients. The axiomatic framework A1-A4 is connected with the framework of standard interference functions [3]. The details 
about the relationship between the model $A 1$ to $A 4$ and Yates' standard interference functions were discussed in [7]. For the purpose of this paper it is sufficient to be aware that there exists a connection between these two models and the results of this paper are applicable to standard interference functions.

\section{REFERENCES}

[1] S. Boyd and L. Vandenberghe, Convex Optimization. New York: Cambridge Univ. Press, 2004

[2] Z.-Q. Luo and W. Yu, "An introduction to convex optimization for communications and signal processing," IEEE J. Sel. Areas Commun., vol. 24, no. 8, pp. 1426-1438, Aug. 2006.

[3] R. D. Yates, "A framework for uplink power control in cellular radio systems," IEEE J. Sel. Areas Commun., vol. 13, no. 7, pp. 1341-1348, 1995.

[4] C. Huang and R. Yates, "Rate of convergence for minimum power assignment algorithms in cellular radio systems," Baltzer/ACM Wireless Netw., vol. 4, pp. 223-231, 1998.

[5] M. Schubert and H. Boche, "QoS-based resource allocation and transceiver optimization," in Foundations and Trends in Communications and Information Theory. Delft, The Netherlands: NOW, 2006, vol. 2.

[6] K. K. Leung, C. W. Sung, W. S. Wong, and T. Lok, "Convergence theorem for a general class of power-control algorithms," IEEE Trans. Commun., vol. 52, no. 9, pp. 1566-1574, Sep. 2004.

[7] H. Boche and M. Schubert, "A unifying approach to interference modeling for wireless networks," IEEE Trans. Signal Process., vol. 58, no. 6, pp. 3282-3297, Jun. 2010.

[8] M. Bengtsson and B. Ottersten, "Optimal and suboptimal transmit beamforming," in Handbook of Antennas in Wireless Communications. Boca Raton, FL: CRC Press, Aug. 2001, ch. 18.

[9] D. Hammarwall, M. Bengtsson, and B. Ottersten, "On downlink beamforming with indefinite shaping constraints," IEEE Trans. Signal Process., vol. 54, no. 9, pp. 3566-3580, Sep. 2006.

[10] A. Wiesel, Y. C. Eldar, and S. S. Shitz, "Linear precoding via conic optimization for fixed MIMO receivers," IEEE Trans. Signal Process., vol. 54, no. 9, pp. 3566-3580, Sep. 2006.

[11] S. Ulukus and R. Yates, "Adaptive power control and MMSE interference suppression," ACM Wireless Netw., vol. 4, no. 6, pp. 489-496, 1998.

[12] N. Vucic and H. Boche, "A tractable method for chance-constrained power control in downlink multiuser MISO systems with channel uncertainty," IEEE Signal Process. Lett., vol. 16, no. 5, pp. 346-349, May 2009.

[13] S. A. Vorobyov, A. B. Gershman, and Z.-Q. Luo, "Robust adaptive beamforming using worst-case performance optimization: A solution to the signal mismatch problem," IEEE Trans. Signal Process., vol. 51, no. 2, pp. 313-324, Feb. 2003.

[14] W. Yu and T. Lan, "Transmitter optimization for the multi-antenna downlink with per-antenna power constraints," IEEE Trans. Signal Process., vol. 55, no. 6, pp. 2646-2660, Jun. 2007.

[15] H. Boche and M. Schubert, "Concave and convex interference functions-general characterization with applications," IEEE Trans. Signal Process., vol. 56, no. 10, pp. 4951-4965, Oct. 2008.

[16] E. Jorswieck, E. Larsson, and D. Danev, "Complete characterization of the Pareto boundary for the MISO interference channel," IEEE Trans. Signal Process., vol. 56, no. 10-2, pp. 5292-5296, Oct. 2008.

[17] T. Alpcan, T. Basar, and S. Dey, "A power control game based on outage probabilities for multicell wireless data network," IEEE Trans. Wireless Commun., vol. 5, no. 4, pp. 890-899, Apr. 2006.

[18] N. Bambos, S. Chen, and G. Pottie, "Channel access algorithms with active link protection for wireless communication networks with power control," IEEE/ACM Trans. Netw., vol. 8, no. 5, pp. 583-597, Oct. 2000.

[19] M. Xiao, N. Shroff, and E. Chong, "A utility-based power-control scheme in wireless cellular systems," IEEE/ACM Trans. Netw., vol. 11, no. 2, pp. 210-221, April 2003.

[20] A. Koskie and Z. Gajic, "A Nash game algorithm for SIR-based power control for 3G wireless CDMA networks," IEEE/ACM Trans. Netw., vol. 13, no. 5, pp. 1017-1026, Oct. 2005.

[21] H. Boche and M. Schubert, "The structure of general interference functions and applications," IEEE Trans. Inf. Theory, vol. 54, no. 11, pp. 4980-4990, Nov. 2008.
[22] H. Boche and M. Schubert, "Concave and convex interference functions-General characterization and applications," IEEE Trans. Signal Process., vol. 56, no. 10, pp. 4951-4965, Oct. 2008.

[23] H. Boche and S. Naik, "Impact of interference coupling-loss of convexity," presented at the IEEE GLOBECOM, Honolulu, HI, Dec. 2009.

[24] C. W. Sung, "Log-convexity property of the feasible SIR region in power-controlled cellular systems," IEEE Commun. Lett., vol. 6, no. 6, pp. 248-249, 2002.

[25] D. Catrein, L. A. Imhof, and R. Mathar, "Power control, capacity, and duality of uplink and downlink in cellular CDMA systems," IEEE Trans. Commun., vol. 52, no. 10, pp. 671-687, 2004.

[26] H. Boche and S. Stanczak, "Convexity of some feasible QoS regions and asymptotic behavior of the minimum total power in CDMA systems," IEEE Trans. Commun., vol. 52, no. 12, pp. 2190-2197, Dec. 2004.

[27] S. Stanczak, M. Wiczanowski, and H. Boche, Theory and Algorithms for Resource Allocation in Wireless Networks, ser. Lecture Notes in Computer Science (LNCS). Berlin, Germany: Springer-Verlag, 2006.

[28] M. Chiang, C. W. Tan, D. Palomar, D. O'Neill, and D. Julian, "Power control by geometric programming," IEEE Trans. Wireless Commun., vol. 6, no. 7, pp. 2640-2651, Jul. 2007.

[29] D. Julian, M. Chiang, D. O'Neill, and S. Boyd, "Qos and fairness constrained convex optimization of resource allocation for wireless cellular and ad hoc networks," in Proc. IEEE INFOCOM, 2002, pp. 477-486.

[30] M. Chiang, "Geometric programming for communication systems," Foundations and Trends in Communications and Information Theory, vol. 2, no. 1/2, 2005.

[31] M. Chiang, P. Hande, T. Lan, and C. W. Tan, "Power control in wireless cellular networks," Foundation and Trends in Networking, vol. 2, no. 4, 2008.

[32] C. W. Sung, "Achieving log-utility fairness in CDMA systems via majorization," IEEE Commun. Lett., vol. 13, no. 9, pp. 625-627, Sep. 2009.

[33] C. W. Tan, D. P. Palomar, and M. Chiang, "Exploiting hidden convexity for flexible and robust allocation in cellular networks," in Proc. IEEE INFOCOM, 2007, pp. 967-972.

[34] S. Stanczak and H. Boche, "On the convexity of feasible QoS regions," IEEE Trans. Inf. Theory, vol. 53, no. 2, pp. 779-783, Feb. 2007.

[35] D. Tse, P. Viswanath, and L. Zheng, "Diversity-multiplexing tradeoff in multiple-access channels," IEEE Trans. Inf. Theory, vol. 50, no. 9, pp. 1859-1874, Sep. 2004.

[36] H. Boche and M. Schubert, "A calculus for log-convex interference functions," IEEE Trans. Inf. Theory, vol. 54, no. 12, pp. 5469-5490, Dec. 2008.

[37] H. Boche, S. Naik, and T. Alpcan, General utility modeling for wireless systems [Online]. Available: http://www.mk.tu-berlin.de/publikationen/objects/2009/gum2009/base_view

[38] J. G. Proakis, Digital Communications, 4th ed. New York: McGrawHill, 0072321113.

[39] H. Boche and M. Schubert, "Complete characterization of the Pareto boundary of interference coupled wireless systems with power constraints-The log-convex case," presented at the Int. Conf. Acoust., Speech, Signal Process. (ICASSP), Taipeh, Taiwan, R.O.C., Apr. 2009.

[40] P. M. Pardalos and S. Vavasis, "Open questions in complexity theory for numerical optimization," Math. Programm., vol. 57, pp. 337-339, 1992.

[41] Z.-Q. Luo and S. Zhang, "Duality gap estimation and polynomial time approximation for optimal spectrum management," IEEE Trans. Signal Process., vol. 57, no. 7, pp. 2675-2689, Jul. 2009.

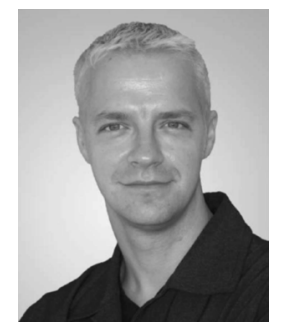

Holger Boche (M'04-SM'07-F'11) received the Dipl.-Ing. and Dr.-Ing. degrees in electrical engineering from the Technische Universität Dresden, Dresden, Germany, in 1990 and 1994, respectively. He graduated in mathematics from the Technische Universität Dresden in 1992., and from 1994 to 1997, he did postgraduate studies in mathematics at the Friedrich-Schiller Universität Jena, Jena, Germany. He received the Dr.rer.nat. degree in pure mathematics from the Technische Universität Berlin, Berlin, Germany, in 1998.

In 1997, he joined the Heinrich-Hertz-Institut (HHI) für Nachrichtentechnik Berlin, Berlin, Germany. Since 2002, he has been a Full Professor for mobile communication networks with the Institute for Communications Systems, Technische Universität Berlin. In 2003, he became Director of the Fraunhofer German-Sino Lab for Mobile Communications, Berlin, Germany and since 
2004 he has also been Director of the Fraunhofer Institute for Telecommunications (HHI), Berlin, Germany. Since October 2010, he has been with the Institute of Theoretical Information Technology and Full Professor at the Technische Universität München, Munich, Germany. He was a Visiting Professor with the ETH Zurich, Zurich, Switzerland, during the 2004 and 2006 winter terms and with KTH Stockholm, Stockholm, Sweden, during the 2005 summer term.

Prof. Boche is a Member of IEEE Signal Processing Society SPCOM and SPTM Technical Committee. Hewas elected a Member of the German Academy of Sciences (Leopoldina) in 2008 and of the Berlin Brandenburg Academy of Sciences and Humanities in 2009. He received the Research Award Technische Kommunikation from the Alcatel SEL Foundation in October 2003, the Innovation Award from the Vodafone Foundation in June 2006 and the Gottfried Wilhelm Leibniz Prize from the Deutsche Forschungsgemeinschaft (German Research Foundation) in 2008. He was corecipient of the 2006 IEEE Signal Processing Society Best Paper Award and recipient of the 2007 IEEE Signal Processing Society Best Paper Award.

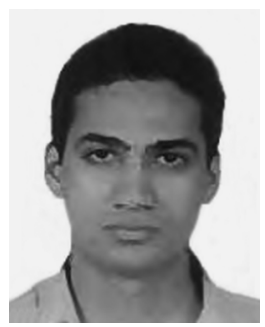

Siddharth Naik received the M.S. degree in electrical engineering with a specialization in wireless systems from the Royal Institute of Technology (KTH), Stockholm, Sweden, in 2004.

He was with the SPANN Lab, IIT Bombay, Mumbai, India, in 2004 and 2005. Since, October 2005 he has been the Fraunhofer German-Sino Lab for Mobile Communications, Berlin, Germany. Since January 2008, he has been a Research Associate at the Technical University of Berlin, Berlin, Germany. Since October 2010, he has been Research Associate with the Institute of Theoretical Information Technology at the Technical University of Munich, Munich, Germany. His research interests are game theory, mechanism design, probability theory and optimization.

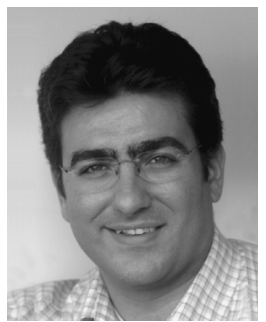

Tansu Alpcan (S'98-M'06) received the B.S. degree in electrical engineering from Bogazici University, Istanbul, Turkey, in 1998 and the M.S. and Ph.D. degrees in electrical and computer engineering from the University of Illinois at Urbana-Champaign (UIUC) in 2001 and 2006, respectively.

He worked as a Senior Research Scientist in Deutsche Telekom Laboratories, Berlin, Germany, between 2006 and 2009. He is currently Assistant Professor (junior professor) in the Technical University Berlin while continuing his affiliation with Deutsche Telekom Laboratories. He is the (co-)author of more than 90 journal and conference papers as well as the book Network Security: A Decision and Game Theoretic Approach (Cambridge Univ. Press, to appear in 2011). His research involves applications of distributed decision making, game theory, and control to various security and resource allocation problems in complex and networked systems.

Dr. Alpcan has received the Fulbright scholarship in 1999 and Best Student Paper Award in the IEEE Conference on Control Applications in 2003. He received the Robert T. Chien Research Award from the UIUC Department of Electrical and Computer Engineering and the Ross J. Martin Research Award from the UIUC College of Engineering in 2006. He was an Associate Editor for the IEEE Conference on Control Applications (CCA) in 2005 and has been TPC member of several conferences, including IEEE INFOCOM from 2007 to 2009. He was the Co-Chair of the Workshop on Game Theory in Communication Networks (GameComm) 2008 and publicity chair of GameNets 2009. He is Steering Board Member and General Chair of the Conference on Decision and Game Theory for Security $(\mathrm{GameSec})$ 2010. He received the Best Paper Award in the 2010 IEEE International Conference on Communications (ICC), Communications and Information Systems Security Symposium. 